Article

\title{
De Novo Assembly and Analysis of Tartary Buckwheat (Fagopyrum tataricum Garetn.) Transcriptome Discloses Key Regulators Involved in Salt-Stress Response
}

\author{
Qi Wu ${ }^{1,2,3, *,+}$, Xue Bai ${ }^{1,2,3,+}$, Wei Zhao ${ }^{3}$, Dabing Xiang ${ }^{1,2,3}$, Yan Wan ${ }^{1,2,3}$, Jun Yan ${ }^{1,2,3}$, \\ Liang Zou ${ }^{1,2,3}$ and Gang Zhao 1,2,3,* \\ 1 Key Laboratory of Coarse Cereal Processing, Ministry of Agriculture, Chengdu 610106, China; \\ baixue@cdu.edu.cn (X.B.); xiangdabing@cdu.edu.cn (D.X.); wanyanyanbest@126.com (Y.W.); \\ yanjun62@cdu.edu.cn (J.Y.); zouliang@cdu.edu.cn (L.Z.) \\ 2 National Research and Development Center for Coarse Cereal Processing, Chengdu 610106, China \\ 3 College of Pharmacy and Biological Engineering, Chengdu University, Chengdu 610106, China; \\ zhaowei6030@126.com \\ * Correspondence: jerviswuqi@126.com (Q.W.); zhaogang@cdu.edu.cn (G.Z.) \\ + These authors have contributed equally to this work.
}

Received: 14 August 2017; Accepted: 15 September 2017; Published: 3 October 2017

\begin{abstract}
Soil salinization has been a tremendous obstacle for agriculture production. The regulatory networks underlying salinity adaption in model plants have been extensively explored. However, limited understanding of the salt response mechanisms has hindered the planting and production in Fagopyrum tataricum, an economic and health-beneficial plant mainly distributing in southwest China. In this study, we performed physiological analysis and found that salt stress of $200 \mathrm{mM} \mathrm{NaCl}$ solution significantly affected the relative water content (RWC), electrolyte leakage (EL), malondialdehyde (MDA) content, peroxidase (POD) and superoxide dismutase (SOD) activities in tartary buckwheat seedlings. Further, we conducted transcriptome comparison between control and salt treatment to identify potential regulatory components involved in F. tataricum salt responses. A total of 53.15 million clean reads from control and salt-treated libraries were produced via an Illumina sequencing approach. Then we de novo assembled these reads into a transcriptome dataset containing 57,921 unigenes with $\mathrm{N} 50$ length of $1400 \mathrm{bp}$ and total length of $44.5 \mathrm{Mb}$. A total of 36,688 unigenes could find matches in public databases. GO, KEGG and KOG classification suggested the enrichment of these unigenes in 56 sub-categories, $25 \mathrm{KOG}$, and 273 pathways, respectively. Comparison of the transcriptome expression patterns between control and salt treatment unveiled 455 differentially expressed genes (DEGs). Further, we found the genes encoding for protein kinases, phosphatases, heat shock proteins (HSPs), ATP-binding cassette (ABC) transporters, glutathione S-transferases (GSTs), abiotic-related transcription factors and circadian clock might be relevant to the salinity adaption of this species. Thus, this study offers an insight into salt tolerance mechanisms, and will serve as useful genetic information for tolerant elite breeding programs in future.
\end{abstract}

Keywords: Fagopyrum tataricum; salt stress; transcriptome; Illumina sequencing; expression analysis

\section{Introduction}

Soil salinization has been an afflicting factor for plant agriculture [1]. More than 800 million hectares of land are suffering from salinization throughout the world [2]. Breeding and application of salt-tolerant elites for each food crop is becoming extremely urgent to feed the global population in future decades. Prior to achieving this goal there should be a good knowledge of the fundamental 
mechanisms of plant salt responses at the molecular, cellular, and whole plant levels. Recently, much progress was made especially in model plants (such as Arabidopsis thaliana and Oryza sativa), improving our understanding of this process. For salinity adaption, plants have generated a complex of strategies mainly containing osmotic tolerance, detoxification of ions, and tolerance of tissue to accumulated ions [2]. Thus, a series of signal perception, signal transduction, transcriptional regulation and biochemical reactions are employed to achieve the responses [3]. Protein kinases (e.g., calcium-dependent protein kinases (CDPKs) and CBL-interacting protein kinases (CIPKs)) are very important for hyperosmotic signal transduction to downstream gene transcription regulation $[3,4]$. Transmembrane transporters, such as $\mathrm{H}^{+}$-ATPase, $\mathrm{Na}^{+} / \mathrm{H}^{+}$exchangers or $\mathrm{Na}^{+} / \mathrm{H}^{+}$antiporters and $\mathrm{Na}^{+} / \mathrm{K}^{+}$cation transporters, are critical for ion homeostasis [1,3]. Physiological evidences demonstrated accumulation of osmolytes (e.g., proline, glycine betaine and mannitol), activation of antioxidant enzymes (e.g., catalase, superoxide dismutase and peroxidase) were utilized to minimize the damages for plant cells [5]. Furthermore, plant hormones (e.g., abscisic acid (ABA), brassinosteroid (BR) and gibberellic acid (GA)) are highly associated with various stress signaling cascades and adaption [6]. Combination of genetic studies and transcriptome investigation has disclosed that many transcription factors are involved in salt stress responses [7-18]. MYB [9], dehydration-responsive element-binding proteins (DREB) [19], basic-domain leucine-zipper (bZIP) [20], CRT/DRE-binding factor (CBF) [21], WRKY [10,12], and NAC [22] were showed to regulate the stress-responsive genes. Nevertheless, understanding of the complexity of salt stress tolerance mechanism in plant deserves more in-depth knowledge.

Recently, next-generation sequencing (NGS) technologies have revolutionized the genomic and transcriptomic studies due to its high accuracy, throughput and sensitivity [23]. At present, NGS-based RNA sequencing (RNA-seq) has been widely employed to decipher various abiotic stress responses in many cases, especially for the species without validly annotated genome [24].

Tartary buckwheat (Fagopyrum tataricum Garetn.) is becoming highly attractive because of the high-quality proteins and pharmaceutical ingredients, such as rutin, quercetin and isoquercetin in the seeds [25]. In China, tartary buckwheat is mainly distributed in the marginal land of Sichuan province, Guizhou province and Yunnan province as the main food of the minorities [26]. However, in recent years, owing to water deficit and irrational irrigation in these arid areas, soil salinization has become a growing obstacle for tartary buckwheat planting. Therefore, dissecting the underlying salt stress mechanisms of $F$. tataricum and subsequent application is of great significance for protecting F. tataricum from yield loss attributing to soil salinization. Several genes related to salt response of buckwheat have been reported [27]. However, a comprehensive understanding of the salt adaption mechanism still remains limited in buckwheat.

In this study, we conducted a genome-wide transcriptome analysis of F. tataricum via an Illumina paired-end sequencing platform. Sequencing and annotation of the F. tataricum genome [28] was undergoing, thus we de novo assembled a transcriptome and carried out global expression profiling to identify the potential regulators involved in salt stress response. This study will provide a valuable source for updating our understanding of the salt tolerance regulatory network in F. tataricum.

\section{Material and Methods}

\subsection{Plant Materials and Growth Conditions}

Tatary buckwheat (F. tataricum cv. Chuanqiao No.1) was used as the experimental materials in this study. The seeds were provided by the National Research and Development Center for Coarse Cereal Processing, Chengdu, Sichuan Province, China. The seeds were sown and grown in small pots (12 cm in diameter and $10 \mathrm{~cm}$ in depth) at the density of 40 seeds for each pot. After germination, the seedlings were cultured in a growth chamber under controlled conditions: $23^{\circ} \mathrm{C}, 12 \mathrm{~h}$ for dark, and $25^{\circ} \mathrm{C}, 12 \mathrm{~h}$ for light with light intensity at $100 \mathrm{umol}^{-2} \cdot \mathrm{s}^{-1}$. The humidity was about $65 \%$ with watering supplied every three days. After growth for two weeks, the seedlings were subjected to 
salinity treatment by supplying with $200 \mathrm{mM}$ sodium chloride solution. Three independent biological replicates with eight seedling plants per replicate for each sample were collected at $0 \mathrm{~h}$ and $24 \mathrm{~h}$ after salinity treatment. The $0 \mathrm{~h}$ (control) and $24 \mathrm{~h}$ (salt treatment) samples were used for physiological analysis and total RNA extraction.

\subsection{Physiological Analysis}

The relative water content (RWC) was measured according to the method of Li et al. [29], and the electrolyte leakage (EL) was measured according to the protocol described by Ishitani [30]. The malondialdehyde (MDA) content, peroxidase (POD) and superoxide dismutase (SOD) activities were tested by the methods used in previous study [31].

\subsection{RNA Extraction and cDNA Library Preparation}

Total RNA of three control and three salt treated biological replicates was extracted using TaKaRa MiniBEST Universal RNA Extraction Kit (TaKaRa, Shiga, Japan) according to the manufacturer's protocols. The integrity and quantity of total RNA samples were assessed by using $1 \%$ agarose gel electrophoresis, Qubit RNA HS Assay Kit (Thermo Fisher Scientific, Waltham, MA, USA) and 2100 Bioanalyzer RNA 6000 Nano Assay Kit (Agilent Technologies, Singapore). mRNA was isolated from 5 ug total RNA (RNA Integrity Number (RIN) $>7.0$, pooled in equal quantity from the total RNA of three replicates) of each sample by magnetic oligo(dT) beads, fragmented into short sequences, and then subjected to complementary DNA (cDNA) library construction by using Illumina TrueSeq RNA library method according to TrueSeq RNA Sample Preparation guide (Illumina Technologies, San Diego, CA, USA).

\subsection{Illumina Sequencing and Sequences Assembly}

The cDNA libraries of control and salt treatment samples were sequenced using Illumina Hiseq 2500 platform (Illumina Technologies) to generate $150 \mathrm{bp}$ paired-end reads. The unqualified reads, including (1) adaptors contaminated reads; (2) unknown nucleotides more than $5 \%$ reads and (3) reads of which more than $50 \%$ of bases showed a $q$-value less than 5 , were removed from the raw reads to obtain clean reads. Trinity program [32] was used to de novo assemble high-quality clean reads into transcriptome. The longest sequences of redundant and chimeric transcripts were defined as unigenes, and then were subjected to downstream functional annotation and coding sequence (CDS) prediction.

\subsection{Coding Sequence Prediction, Functional Annotation and Classification of Unigenes}

For CDS prediction, unigenes were compared with GenBank NR (NCBI non-redundant protein) [33] and Swiss-Prot databases [34] by BLAST [35] (E-value cut-off $=1 \times 10^{-5}$ ), and the best alignment was used to determine the $5^{\prime}-3^{\prime}$ orientation and the putative coding sequence region. ESTScan 3.0.3 [36] was used to predict the putative open reading frame (ORF) region of the unigenes without alignment in GenBank NR and Swiss-Prot databases.

To annotate the unigenes dataset, the sequences were aligned against GenBank NR [33], GenBank NT (NCBI nucleotide sequences) [33], Swiss-Prot databases [34] and KOG (euKaryotic Orthologous Groups) [37] using NCBI BLAST 2.2.28+ [35] with an E-value threshold of $1 \times 10^{-5}$. Meanwhile, these unigenes were also annotated by KEGG (Kyoto Encyclopedia of Genes and Genomes) [38] using KAAS (E-value cut-off $=1 \times 10^{-10}$ ) [39], by GO (Gene Ontology) [40] using Blast2GO v2.5 (E-value cut-off $=1 \times 10^{-6}$ ) [41] and by Pfam (Protein family) [42] using HMMER 3.0 [43] (E-value cut-off $=0.01$ ). 


\subsection{Identification of Transcription Factor Families}

To identify the potential transcription factor encoding genes in the transcriptome data, the assembled unigenes sequences were analyzed using PlantTFcat online tool (http://plantgrn. noble.org/PlantTFcat) as previously described [40].

\subsection{Expression Analysis}

The clean reads were mapped onto the assembled unigenes transcriptome using Bowtie [44], and the read count number for each gene was measured by RNA-Seq by Expectation-Maximization (RSEM) [45]. The estimated expression levels of all genes were calculated and normalized to fragments per kilobase of transcript per killion fragments mapped (FPKM) [46]. To identify the differentially expressed genes (DEGs), read count for each gene was normalized with the Trimmed mean of M-values (TMM) method [47], and then DEGseq [48] was used to screen the genes whose $q$-value were less than 0.005 and $\mid \log 2$ FoldChange $\mid>1$ between control and salinity treatment samples. Further, the identified DEGs were subjected to GO enrichment analysis by GOseq based on the Wallenius non-central hypergeometric distribution with the corrected $p$-value less than 0.05 [49]. Moreover, KEGG pathway enrichment analysis of the DEGs was also implemented using KEGG Orthology Based Annotation System (KOBAS) [50].

\subsection{Identification of Simple Sequence Repeats (SSRs)}

MISA (MIcroSAtellite) V1.0 [51] with default parameters was used to analyze distribution of various types of SSRs in all F. tataricum unigenes. The minimum number of repeat units for mono-nucleotide and di-nucleotide repeats were ten and six, respectively, while that for tri-nucleotide, tetra-nucleotide, penta-nucleotide and hexa-nucleotide repeats was five.

\subsection{Reverse Transcription and Real-Time PCR}

Two micrograms of total RNA for each sample were subjected to DNA digestion and reverse transcription to synthesize first-strand cDNA using EasyScript One-Step gDNA Removal and cDNA Synthesis SuperMix Kit (Transgen Biotech, Beijing, China). The cDNA reaction product was diluted 10-fold and $2 \mathrm{uL}$ of the dilution was used to carry out real-time PCR using TransStart Green qPCR SuperMix (Transgen Biotech) on a real-time PCR system (LineGene 9600, BIOER, Hangzhou, China). The real-time PCR program consists of $94{ }^{\circ} \mathrm{C}$ for $5 \mathrm{~min}, 39$ cycles of denaturing at $95{ }^{\circ} \mathrm{C}$ for $10 \mathrm{~s}$, annealing and extension at $59{ }^{\circ} \mathrm{C}$ for $20 \mathrm{~s}$. An actin gene was used as the internal control [52]. The relative expression level of detected genes was quantified by the $2^{-\Delta \Delta \mathrm{Ct}}$ algorithm [37] using Microsoft Excel 2015 (Microsoft, Redmond, WA, USA). For each gene, we performed at least three biological replicates to determine the relative expression level. The related primers used for real-time PCR were list in Table S8.

\subsection{Statistical Analysis}

The data of various physiological indices in Figure 1 were subjected to Student's $t$-test analysis using Microsoft Excel 2015.

\section{Results}

\subsection{Physiological Analysis}

In order to evaluate the effects of salt on physiological activities in F. tataricum seedlings, we measured a series of key physiological indices including relative water content (RWC), electrolyte leakage (EL), malondialdehyde (MDA) content, superoxide dismutase (SOD) and peroxidase (POD) activities. Two-week-old Chuan Qiao No.1 seedlings were treated with $200 \mathrm{mM} \mathrm{NaCl}$ solution, and then 
the aerial parts of seedling plants were collected at $0 \mathrm{~h}$ (control) and $24 \mathrm{~h}$ (salt treatment) after salt treatment. The collected seedling samples were used for physiological analysis.

A
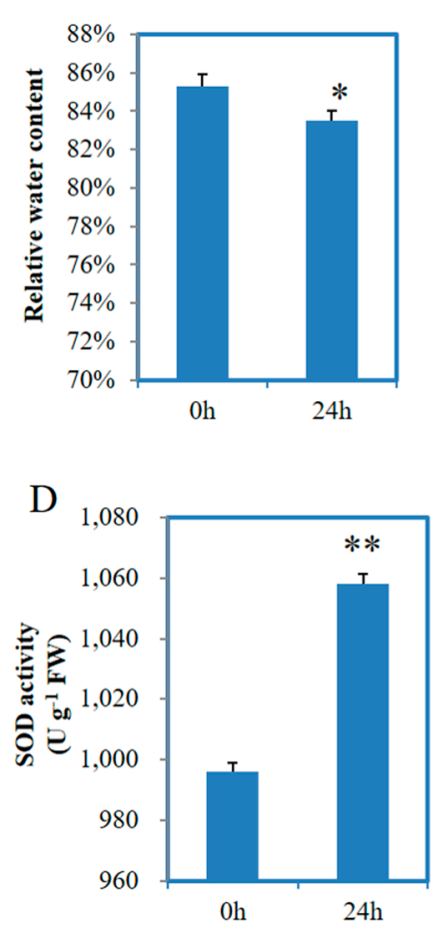

$\mathrm{B}$

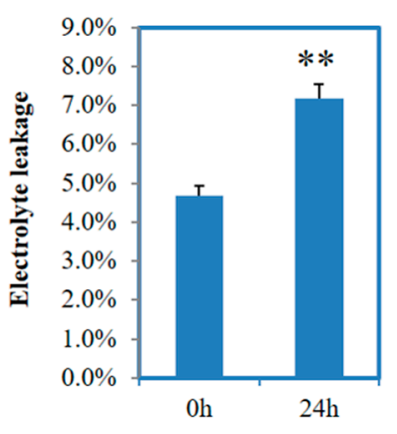

$\mathrm{E}$

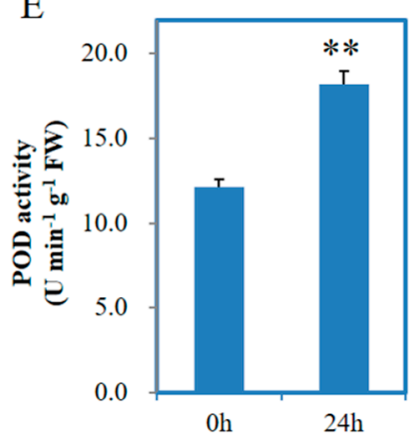

$\mathrm{C}$

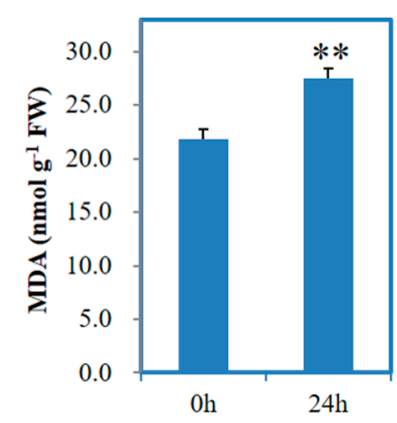

Figure 1. Measurement of relative water content (A); electrolyte leakage (B); malondialdehyde (MDA) (C); superoxide dismutase (SOD) (D) and peroxidase (POD) activities (E) in tartary buckwheat seedlings under control and salt stress conditions. $0 \mathrm{~h}$ and $24 \mathrm{~h}$ represent control and salt treatment conditions. Values are mean $\pm \mathrm{SD}(n=3)$. Single asterisk $\left(^{*}\right)$ and double asterisks $\left.{ }^{(* *}\right)$ stand for $p<0.05$ and $p<0.01$, respectively, determined by student's $t$-test.

As shown in Figure 1A, the RWC in salt stress plants was decreased $(p$-value $<0.05)$ than that in control plants. The EL analysis demonstrated that the EL was significantly increased ( $p$-value $<0.01)$ from $4.7 \%$ to $7.2 \%$ after salt treatment (Figure 1B). The MDA content was sharply increased ( $p$-value $<0.01$ ) by $25.9 \%$ after salt treatment than control (Figure 1C). Meanwhile, we found both SOD and POD activities were significantly increased ( $p$-value $<0.01$ ) under salt stress (Figure 1D,E). These results suggest that salt stress of $200 \mathrm{mM} \mathrm{NaCl}$ solution is able to significantly affect the physiological activities in F. tataricum seedlings.

\subsection{RNA-Seq and De Novo Assembly of the F. tataricum Transcriptome}

To elucidate the underlying salt stress responsive mechanisms in tartary buckwheat, we carried out RNA-seq analysis. Total RNA of control and salt treated samples were extracted, followed by cDNA library construction and paired-end Illumina deep sequencing. After removing the low-quality reads and adaptor sequences from reads, in total we obtain 23.81 million and 29.34 million clean reads from control and salt treatment libraries, which yielded 7.14 billion and 8.80 billion nucleotides, respectively (Table 1). With respect to GC content, control library reached $48.76 \%$ and that of salt treatment library accounted for $46.70 \%$ (Table 1). The percentage of Q20 sequences (with an error probability of 0.01 ) in control library reached $97.85 \%$, while that in salt treatment library was $98.03 \%$ (Table 1). Using Trinity platform [32], we de novo assembled the two libraries clean reads into 75,287 transcripts, from which 57,921 unigenes were generated (Table 1). All the unigenes length ranged from $201 \mathrm{bp}$ to $18,866 \mathrm{bp}$. 
The mean length, median length and N50 length of all assembled unigenes were $768 \mathrm{bp}, 387 \mathrm{bp}$, and $1400 \mathrm{bp}$, respectively (Table 1).

An overview of the unigenes size distribution was showed in Figure 2. Among these unigenes, $23,845(41.17 \%)$ were longer than $500 \mathrm{bp}$, and that longer than $1000 \mathrm{bp}$ reached 13,930 (24.05\%). The assembled unigenes dataset was used as reference transcriptome for further analysis. The reference transcriptome data generated in this study could be found at the Gene Expression Omnibus (GEO), National Centre for Biotechnology Information (NCBI) (https:/ / www.ncbi.nlm.nih.gov/geo/) under the accession number GSE104167.

Table 1. Characteristics of generated read data and assembled Fagopyrum tataricum transcriptome data.

\begin{tabular}{ccc}
\hline Item & CQ_0h & CQ_Na24h \\
\hline Raw reads number & $23,828,556$ & $29,351,455$ \\
Clean reads & $23,816,967$ & $29,340,188$ \\
Base number (Gb) & 7.14 & 8.80 \\
GC content (\%) & $48.76 \%$ & $46.70 \%$ \\
Q20 percentage & $97.85 \%$ & $98.03 \%$ \\
\hline
\end{tabular}

\begin{tabular}{cc}
\hline \multicolumn{2}{c}{ Unigenes } \\
\hline Number of unigenes & 57,921 \\
Min length of unigenes (bp) & 201 \\
Mean length of unigenes (bp) & 768 \\
Max length of unigenes (bp) & 18,866 \\
N50 of unigenes (bp) & 1,400 \\
Total Nucleotides of unigenes (bp) & $44,508,371$ \\
\hline
\end{tabular}

CQ_0h and CQ_Na24h represent control and salt-treated samples respectively.

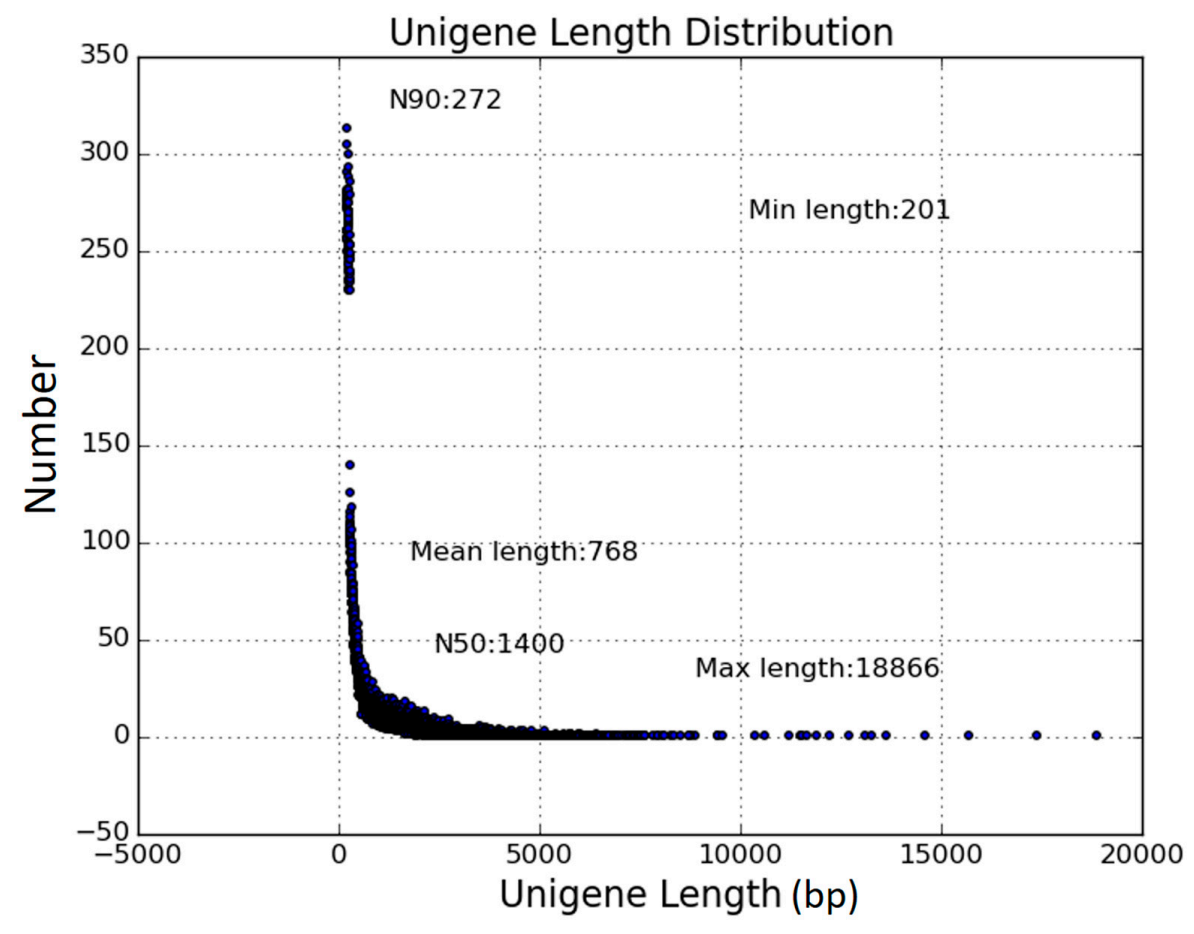

Figure 2. Length distribution of unigenes.

\subsection{Functional Annotation and Classification of Transcriptome Sequences}

In order to fully annotate the unigenes dataset, we aligned each unigene sequence against Genebank NR [33], Genebank NT [33], Pfam [42], KOG [37], Swiss-Prot [34], KEGG [38] and GO [40] databases. Our analysis showed that, out of 57,921 unigenes, 30,500 (52.65\%) were revealed to have 
significant matches in Genebank NR (Table 2), displaying the highest hit rate among all annotation events in the six public databases. The annotated unigenes number in Pfam, Genebank NT and Swiss-Prot were 20,859 (36.01\%), 21,064 (36.36\%) and 23,445 (40.47\%), respectively (Table 2). By using Blast2GO v2.5, we found that 20,834 (35.96\%) matched known proteins in GO (Table 2). The hits similarity in KEGG and KOG were 11,206 (19.34\%) and 11,791 (20.35\%) (Table 2), respectively, indicating relatively lower annotation rate than that in the other four databases. Among all unigenes, $4813(8.3 \%)$ could well match the known proteins in every database and 36,688 (63.34\%) had significant matches in at least one database (Table 2). On the other hand, we found that those with non-annotation in any one of the databases reached 21,233 (36.66\%) (Table 2), which could result from the specific genes in F. tataricum, or the error happened in sequencing. According to the similarity search in Genebank NR, over $20 \%$ of the F. tataricum transcriptome sequences shared strong similarity with genes of Beta vulgaris followed by Vitis vinifera (10.9\%), Arabidopsis thaliana (4.9\%), Theobroma cacao (3.6\%), and Jatropha curcas (3.2\%) (Figure 3).

Table 2. Summary of functional annotation in six public databases.

\begin{tabular}{ccc}
\hline & Number of Unigenes & $\mathbf{\%}$ \\
\hline Annotated in Genebank NR & 30,500 & 52.65 \\
Annotated in Genebank NT & 21,064 & 36.36 \\
Annotated in KEGG & 11,206 & 19.34 \\
Annotated in SwissProt & 23,445 & 40.47 \\
Annotated in Pfam & 20,859 & 36.01 \\
Annotated in GO & 20,834 & 35.96 \\
Annotated in KOG & 11,791 & 20.35 \\
Annotated in all databases & 4813 & 8.3 \\
Annotated in at least one database & 36,688 & 63.34 \\
Not annotated in all database & 21,233 & 36.66 \\
Total Unigenes & 57,921 & 100 \\
\hline
\end{tabular}

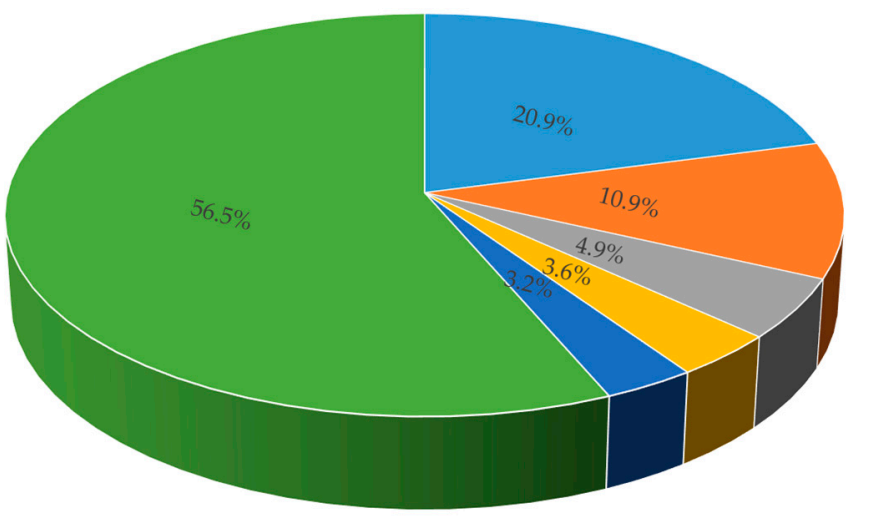

$$
\begin{array}{lll}
\text { - Beta vulgaris } & \text { - Vitis vinifera } & \text { - Arabidopsis thaliana } \\
\text { m Theobroma cacao } & \text { - Jatropha curcas } & \text { m other }
\end{array}
$$

Figure 3. Species distribution of the matched transcriptome sequences in Genebank non redundant (NR) database.

To determine the gene ontology of transcriptome sequences, Blast2GO was used to analyze the predicted protein sequences similarity with Genebank NR and Pfam proteomes. We found $20,834(35.96 \%)$ unigenes could be assigned to 56 GO sub-categories under biological process, cellular component and molecular function categories (Table S1). Among biological process, 'cellular

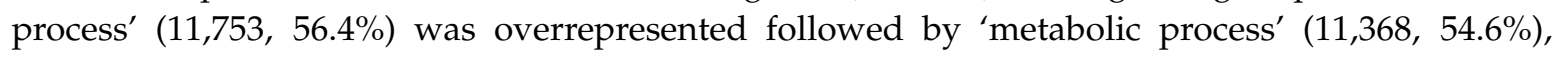


'single-organism process' (8864, 42.5\%), 'biological regulation' (3736, 17.9\%), 'regulation of biological process' $(3477,16.7 \%)$, 'localization' $(3334,16 \%)$, and 'response to stimulus' $(2345,11.3 \%)$ (Figure 4). With respect to cellular component, both 'cell' and 'cell part' $(6399,30.7 \%)$ were the most dominant groups followed by 'macromolecular complex' (4258, 20.4\%), 'organelle' (4150, 19.9\%), 'membrane' (3068, 14.7\%), and 'membrane part' $(2889,13.9 \%)$ (Figure 4). Under molecular function term, 'binding' $(11,891,57.1 \%)$, 'catalytic activity' $(9823,47.1 \%)$, 'transporter activity' $(1374,6.6 \%)$, 'nucleic acid binding transcription factor activity' $(727,3.5 \%)$, 'structural molecule activity' $(601,2.9 \%)$ and 'molecular transducer activity' (369, 1.8\%) were highly represented (Figure 4).

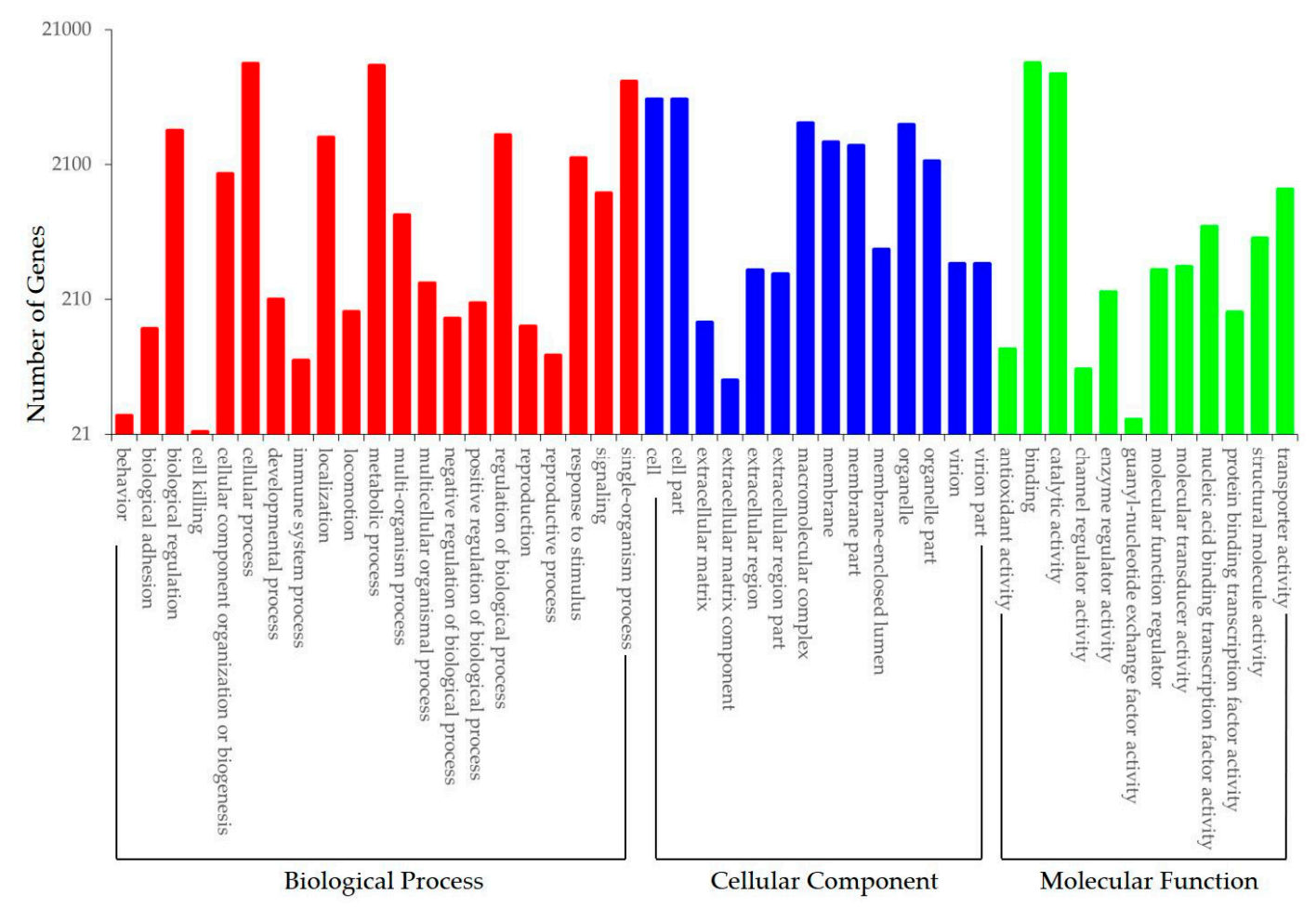

Figure 4. Gene ontology analysis of the Fagopyrum tataricum transcriptome.

Further, KOG classification revealed that 11,791 transcriptome sequences were clustered into 25 functional categories (Table S2). Among the 25 groups, 'General function prediction only' represented the most enriched term $(2049,17.4 \%)$, which was followed by 'Posttranslational modification, protein turnover, chaperones' (1583, 13.4\%), 'Signal transduction mechanisms' (1142, 9.7\%), 'Intracellular trafficking, secretion, and vesicular transport' (790, 6.7\%), 'Translation, ribosomal structure and biogenesis' (769, 6.5\%), 'Carbohydrate transport and metabolism' (663, 5.6\%), 'Energy production and conversion' (588,5.0\%), 'Secondary metabolites biosynthesis, transport and catabolism' (587, 5.0\%), 'RNA processing and modification' (578, 4.9\%) (Figure 5). The smallest group was 'Cell motility' which only contained 4 genes. 


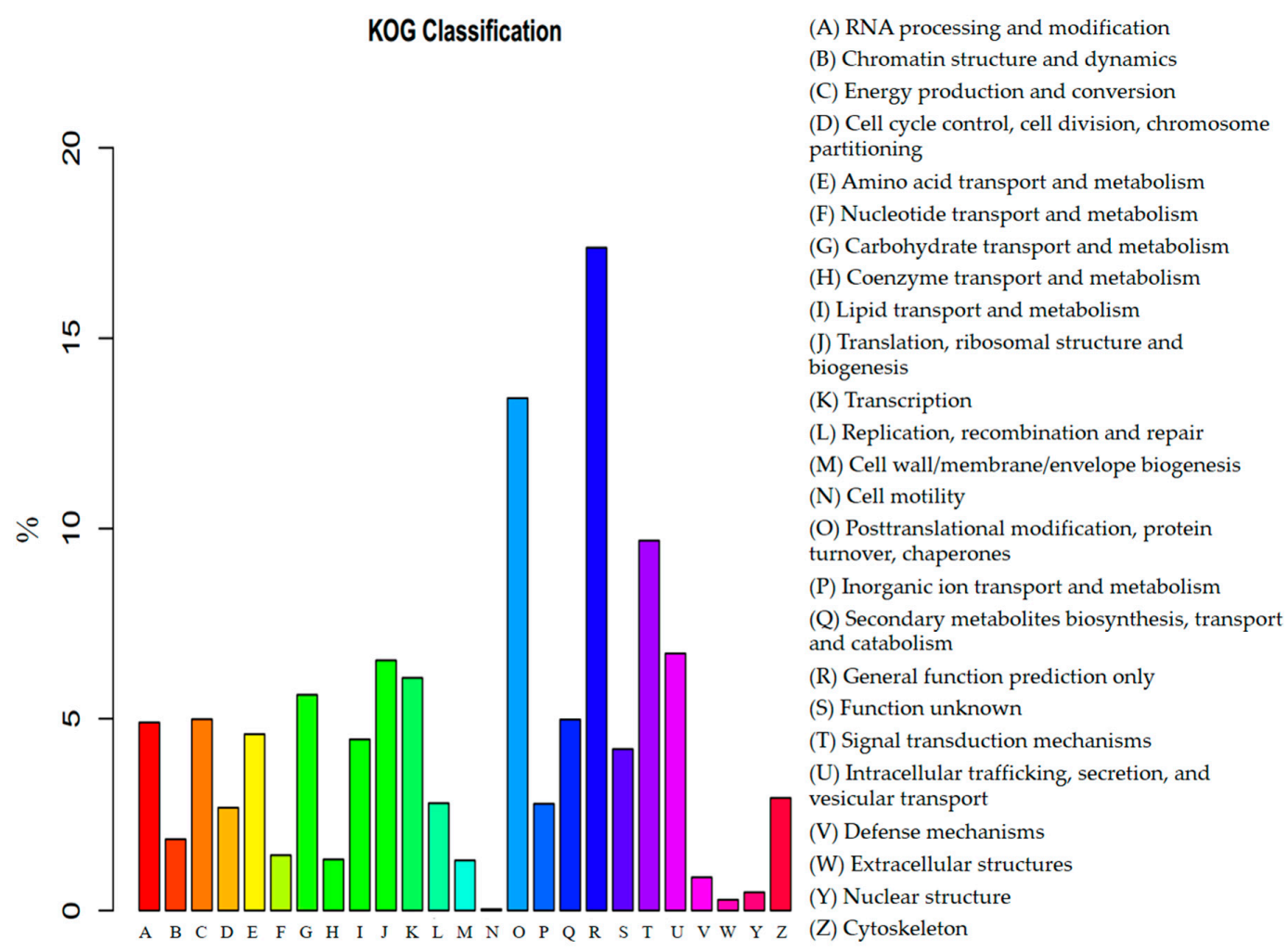

Figure 5. KOG (euKaryotic Orthologous Groups) function classification of the F. tataricum transcriptome.

To better understand the biological functions of our transcriptome genes on the biochemical pathways, we performed KEGG classification by comparing the unigenes with KEGG proteome data using the online KAAS. In total, we obtained 11,206 orthologs involved in 273 pathways in KEGG protein database (Table S3). The most enriched pathways were 'Carbon metabolism' (ko01200), 'Biosynthesis of amino acids' (ko01230), 'Ribosome' (ko03010), 'Plant hormone signal transduction' (ko04075), and 'Protein processing in endoplasmic reticulum' (ko04141). All the annotated unigenes could be categorized into five KEGG functional groups, out of which 'Metabolism' was overrepresented (5811, 51.9\%) (Figure 6). In 'Metabolism', 'Carbon metabolism' (1079, 9.6\%) was highly enriched followed by 'Overview' (797, 7.1\%), 'Energy metabolism' (795, 7.1\%), 'Amino acid metabolism' (725, 6.5\%), and 'Lipid metabolism' (556, 5.0\%) (Figure 6). 2324 unigenes were classified into 'Genetic Information Processing', including 'Translation' (921, 8.2\%), 'Folding, sorting and degradation' (787, 7.0\%), 'Transcription' (363, 3.2\%), and 'Replication and repair' (253, 2.3\%) (Figure 6). In 'Organismal Systems', 2201 unigenes were mainly distributed in 'Endocrine system' (459, 4.1\%), 'Environmental adaptation' (400, 3.6\%), and other sub-categories. In addition, 1,351 unigenes were involved in 'Cellular Processes', in which 'Transport and catabolism' (614, 5.5\%), 'Cell growth and death' (424, 3.8\%), 'Cellular community' (167, 1.5\%), and 'Cell motility' (146, 1.3\%) were highly enriched (Figure 6). 'Environmental Information Processing' accounted for 1269 unigenes, consisted of 'Signal transduction' (1143, 10.2\%), 'Membrane transport' (120,1.1\%), and 'Signaling molecules and interaction' $(6,0.05 \%)$ (Figure 6). Together, these results suggest carbohydrate and amino acid metabolism, various signal transduction and translation events become active in F. tataricum under salt stress. 


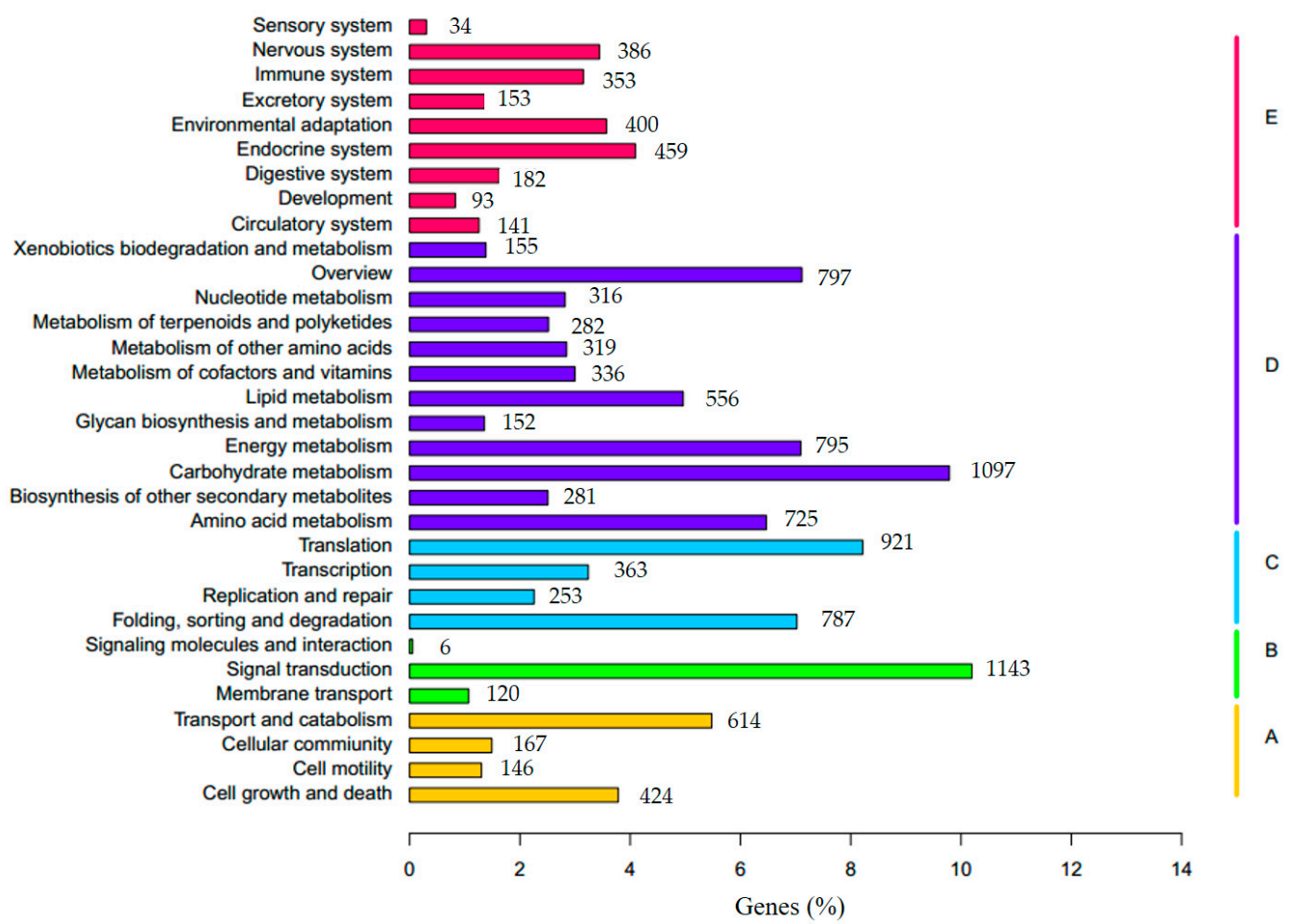

Figure 6. KEGG (Kyoto Encyclopedia of Genes and Genomes) classification of the F. tataricum transcriptome. (A-E) beside the right vertical lines means five different KEGG functional groups. (A) Cellular Processes; (B) Environmental Information Processing; (C) Genetic Information Processing; (D) Metabolism; (E) Organismal Systems.

\subsection{Identification of Transcription Factor Families}

In plants, transcription factors usually play pivotal roles in growth and development processes. Accumulating evidence indicates that many transcription factor families are involved in abiotic stress responses [53]. We predicted the potential transcription factors using PlantTFcat online tool. In total, we identified 93 transcription factor families, including 3107 unigenes, accounting for $5.4 \%$ of the transcriptome (Table S4). Out of 93 families, 'C2H2' (473, 15.2\%) was mostly overrepresented, followed by 'WD40-like' (285, 9.2\%), 'MYB-HB-like' (193, 6.2\%), CCHC (176, 5.7\%), 'PHD' (148, 4.8\%), 'bHLH' (121, 3.9\%), 'AP2-EREBP' (104, 3.4\%), and 'bZIP' (95, 3.1\%).

\subsection{Analysis of Differentially Expressed Genes}

To investigate the DEGs between control and salt treatment libraries, we mapped the clean reads from each sample onto the F. tataricum reference transcriptome using Bowtie. Approximately $81.3 \%$ and $84.7 \%$ of the total high-quality reads from control and salt treatment libraries were mapped to the reference transcriptome, respectively. Using DEGseq, with the cutoff of $q$-value $<0.005$ and $\mid \log 2$ FoldChange $\mid>1$, we obtained 455 DEGs (Figure S1). Among these DEGs, 404 unigenes had higher expression levels under salt treated conditions, whereas only 51 unigenes had higher expression levels under control conditions.

To understand the biological function of the DEGs, we performed GO term enrichment using Blast2GO. Out of 455 DEGs, 363 DEGs were highly abundant in $25 \mathrm{GO}$ terms (Corrected $p$-Value $<0.05$ ). GO enrichment analysis revealed that these DEGs were overrepresented in 'oxidation-reduction process', 'endopeptidase inhibitor activity', 'endopeptidase regulator activity', 'peptidase inhibitor activity', 'peptidase regulator activity', 'cell morphogenesis', and 'cellular component morphogenesis' (Figure 7A and Table S5). Most of the up-regulated DEGs in salt treatment were enriched in 'cell morphogenesis', 'cellular component morphogenesis', 'oxidation-reduction process', 'endopeptidase 
inhibitor activity', 'endopeptidase regulator activity', 'peptidase inhibitor activity', 'peptidase regulator activity', 'cell wall macromolecule metabolic process', and 'oxidoreductase activity' (Table S5). Meanwhile, the down-regulated DEGs under salt treated conditions were overrepresented in 'ATP metabolic process', 'purine nucleoside triphosphate metabolic process', 'ribonucleoside triphosphate metabolic process', 'purine ribonucleoside triphosphate metabolic process', 'nucleoside triphosphate metabolic process' and 'monovalent inorganic cation transport' (Table S5). These results indicated the biological functions described above might be activated under salt stress in F. tataricum.

In addition, in order to further predict which biochemical pathways these DEGs may be involved in, we carried out KEGG enrichment analysis. The results revealed that the DEGs were highly represented in pathway terms 'Antigen processing and presentation' (ko04612), 'Protein processing in endoplasmic reticulum' (ko04141), 'Estrogen signaling pathway' (ko04915), 'Toxoplasmosis' (ko05145), and 'Plant-pathogen interaction' (ko04626) (Figure 7B and Table S6).

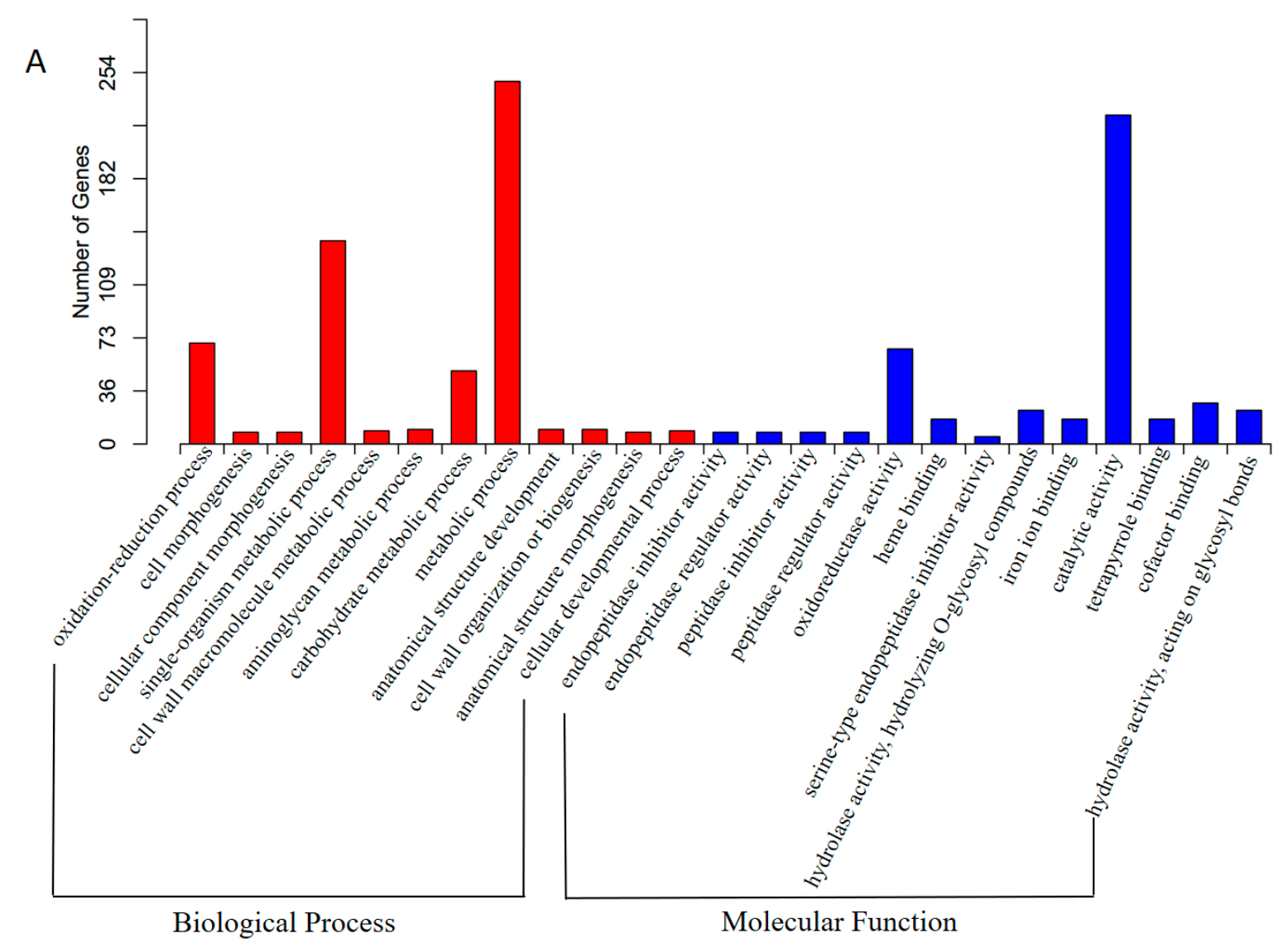

Figure 7. Cont. 


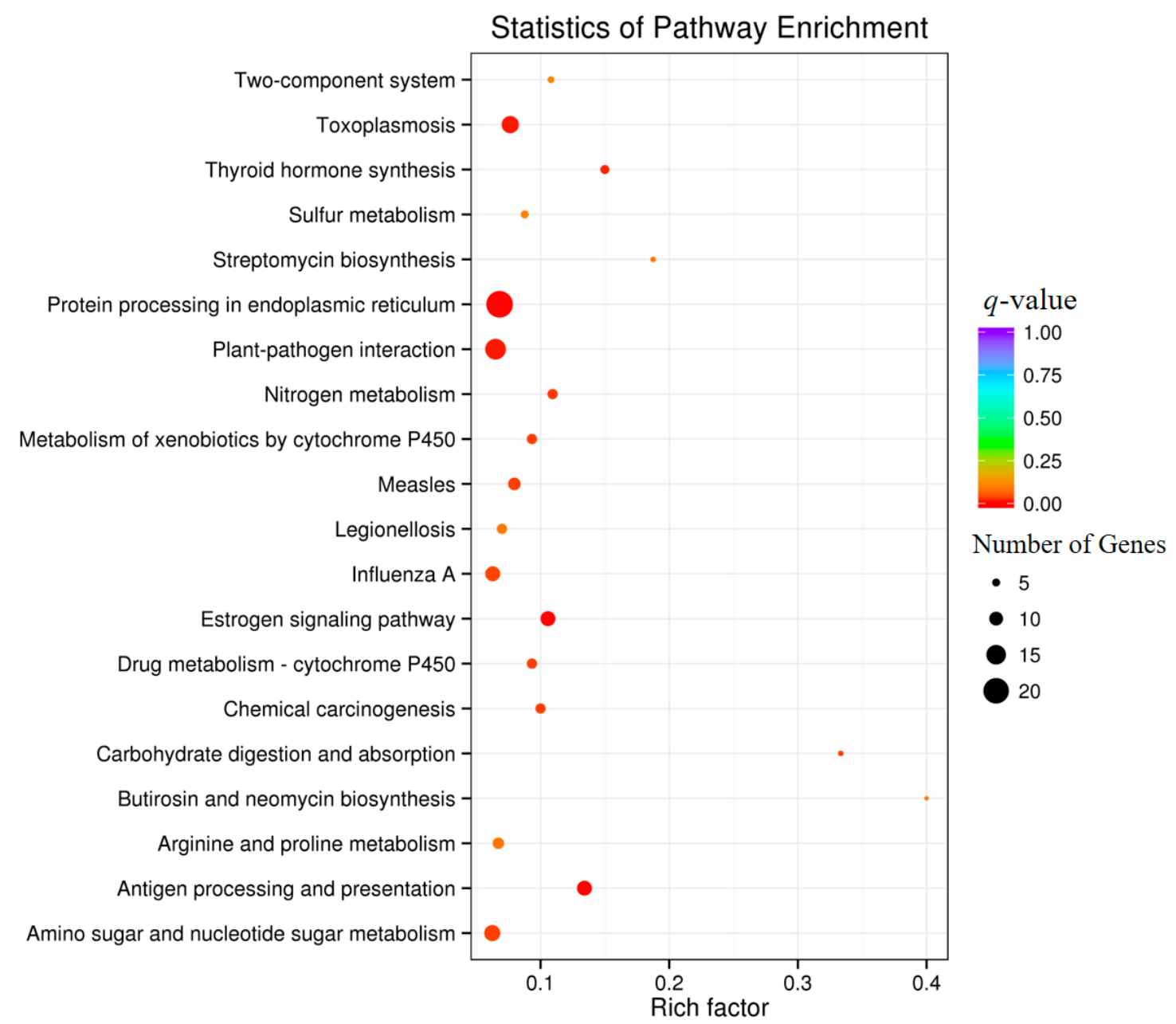

Figure 7. Gene ontology and KEGG analysis of differentially expressed genes (DEGs). (A) GO (gene ontology) classification of the DEGs. BP: GO term type of biological process; MF: GO term type of molecular function; (B) KEGG classification of the DEGs.

\subsection{Validation of Differentially Expressed Genes by Real-Time PCR}

To confirm the reliability of expression profile data obtained by RNA-seq analysis, we conducted real-time PCR analysis. Fourteen unigenes randomly selected from the DEGs were further qualified the expression levels in control and salt treated samples. The fold changes of these genes obtained by real-time PCR were consistent with that obtained by RNA-seq analysis (according to Pearson correlation analysis, $R^{2}=0.9372$ ) (Figure 8 ), thus confirming the accuracy and strong reliability of the expression analysis results. 


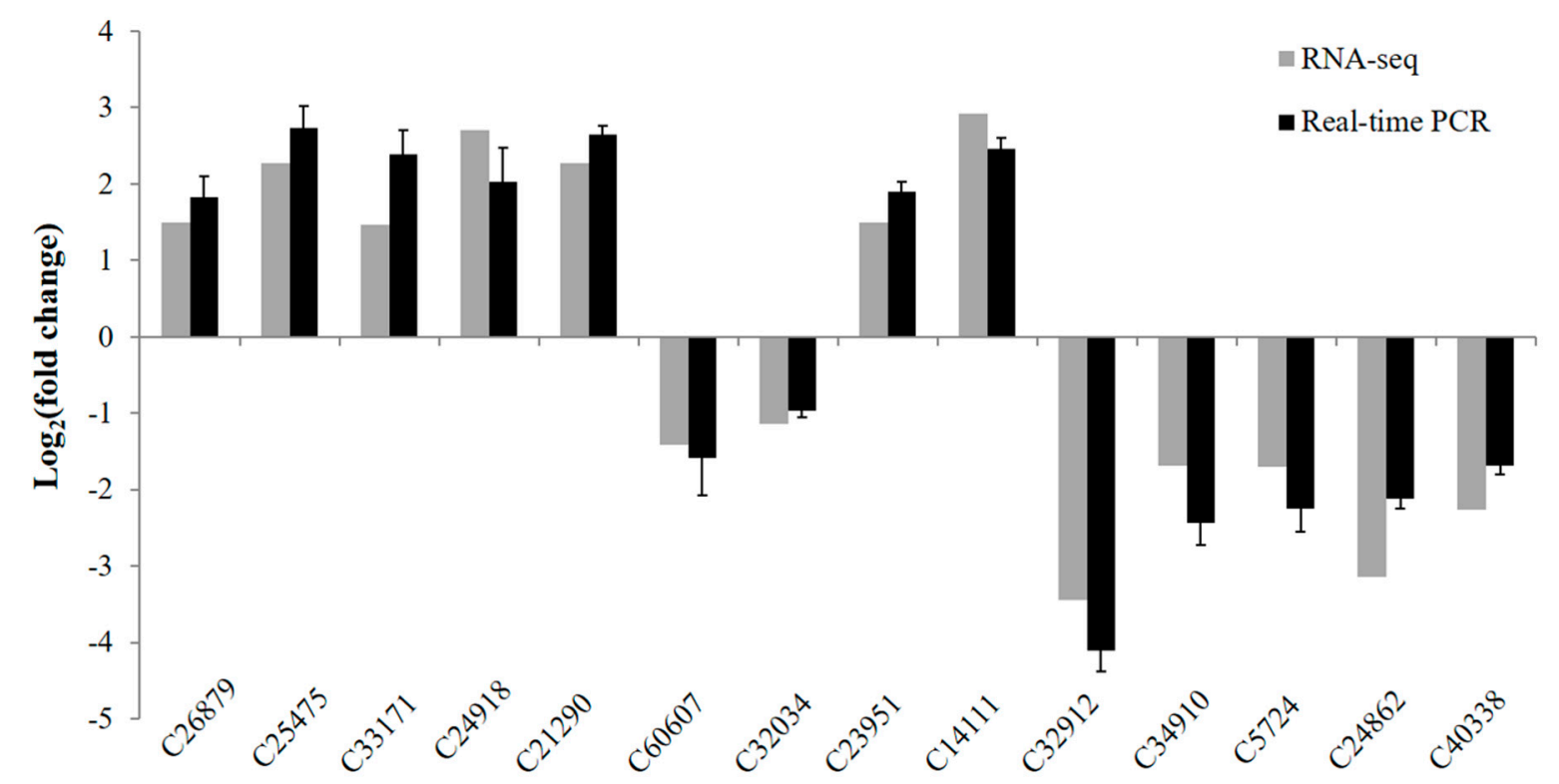

Figure 8. Validation of RNA sequencing (RNA-seq) expression profiles by real-time PCR. The gray bars stand for fold changes based on read count number from normalized RNA-seq data. The black bars stand for fold changes of relative expression levels that were obtained by real-time PCR based on the $2^{-\Delta \Delta \mathrm{Ct}}$ algorithm. Values are mean $\pm \mathrm{SD}(n=3)$.

\subsection{Identification of Simple Sequence Repeats}

Molecular markers, such as Random Amplified Polymorphic DNA (RAPD), Restriction Fragment Length Polymorphism (RFLP), Single Nucleotide Polymorphism (SNP) and Simple Sequence Repeats (SSRs), have been extensively used as powerful tools for genetic study and marker-assisted selection (MAS) in many food crops [54]. In this study, we performed SSRs screening using MISA search tool [51].

By screening 57,921 transcriptome sequences, a total of 6176 potential SSRs were identified to distribute among 5455 unigenes, out of which 599 unigenes were found to harbor more than one SSR. The most abundant SSR type was mononucleotide repeats $(3678,59.6 \%)$, followed by tri-nucleotide $(1410,22.8 \%)$, di-nucleotide $(1010,16.4 \%)$, tetra-nucleotide $(49,0.8 \%)$, hexa-nucleotide (21, 0.3\%), and penta-nucleotide repeats $(8,0.1 \%)$ (Figure 9). Among all SSRs, the most dominant motif was A/T (3641, 59\%), in which 9-12 tandem repeats were overrepresented followed by 13-16, 17-20 and 21-27 tandem repeats. In di-nucleotide repeats, AT/AT displayed the higher frequency (545, 8.8\%) followed by AG/CT $(378,6.1 \%)$. For tri-nucleotide repeats, AAG/CTT showed the most abundant occurrence $(434,7.0 \%)$ followed by ATC / ATG $(218,3.5 \%)$, ACC/GGT $(181,2.9 \%)$, AAC/GTT (125, 2.0\%), and AGG/CCT (120, 1.9\%) (Table S7). Among di-nucleotide and tri-nucleotide repeats, SSRs with 5-8 tandem repeats were the most common tandem repeat. 


\section{Distribution of SSR Motifs}

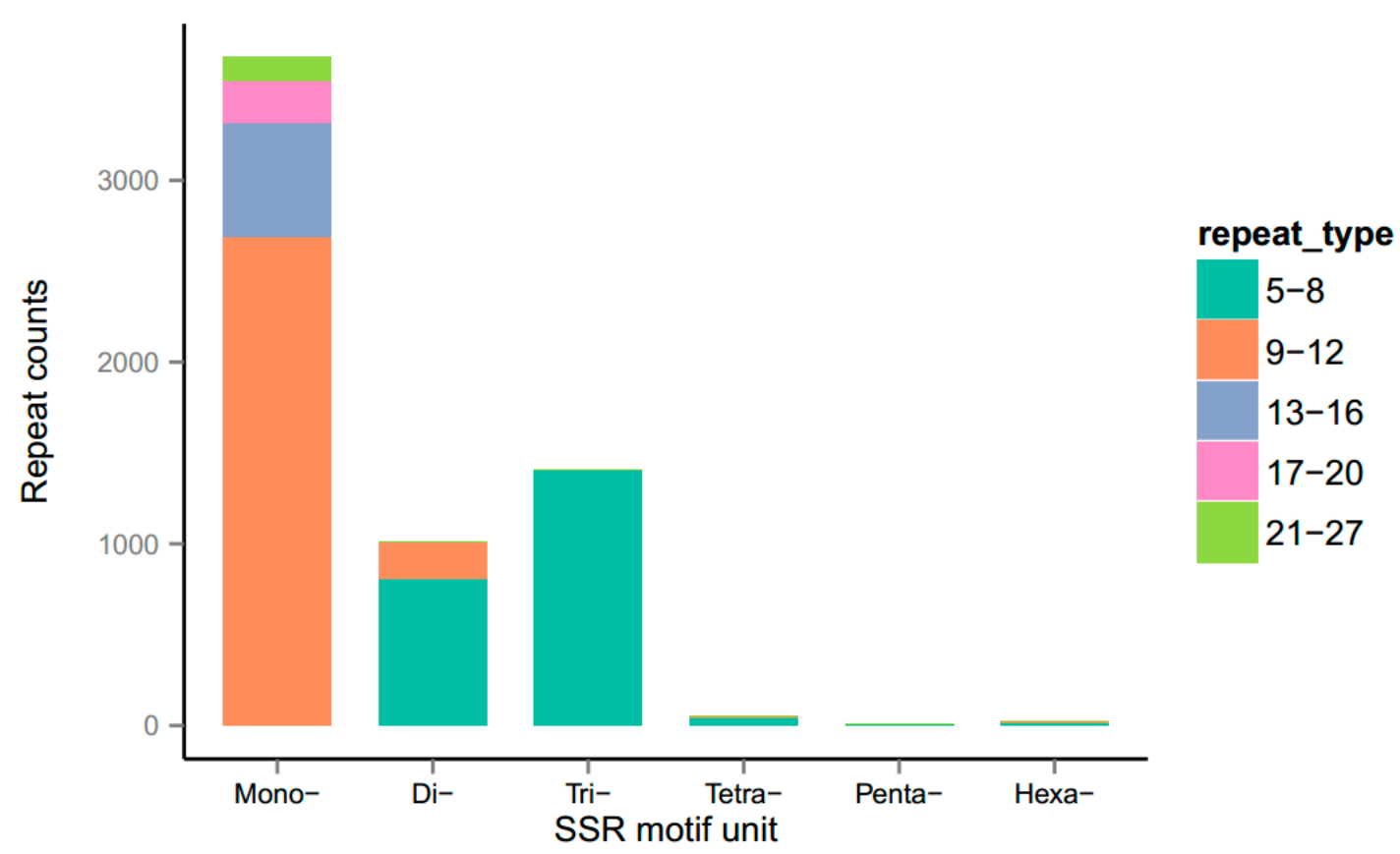

Figure 9. Distribution of simple sequence repeats (SSRs) identified from F. tataricum unigenes.

\section{Discussion}

Soil salinization is a severe threat to the yield of many crops. Recently, transcriptome studies on a large number of species, such as rice, Arabidopsis, smooth cordgrass (Spartina alterniflora) [14], wheat (Triticum aestivum) [15] and Caragana korshinskii [55], have been intensively carried out to decode the salt stress response. These studies have largely improved our understanding of salt-responsive mechanisms of plants. However, more investigation is needed especially for the uncharacterized species. In this study, we characterized the salt-responsive transcriptome of F. tataricum. To our knowledge, before this study, there are seldom reports focused on globally deciphering the salt response via RNA-seq in F. tataricum.

Chuan Qiao No. 1 is one of the most widely grown tartary buckwheat cultivar in Sichuan Province. Considering the importance of seedling stage for tartary buckwheat development and yield, we used two-week-old seedlings as the experimental materials for physiological activities analysis and RNA-seq analysis. By measuring the RWC, we found salt stress could cause water loss in seedling plants at $24 \mathrm{~h}$ after salt treatment. EL and MDA content are usually used to evaluate the integrity of plant cell membranes under abiotic stress [56]. Significantly increased EL and MDA content indicated $200 \mathrm{mM}$ $\mathrm{NaCl}$ solution has largely affected the robust status of cell membrane in tartary buckwheat seedlings. When exposed to abiotic stress, plant antioxidant enzymes such as POD and SOD are usually activated to protect cells and subcellular systems from damage caused by reactive oxygen species (ROS) [57]. Consistently, we tested and found increased POD and SOD activities in seedling plants after salt treatment for $24 \mathrm{~h}$.

Due to unavailable genome information, we de novo assembled a transcriptome of $F$. tataricum. A total of 53.15 million clean reads yielded 57,921 unigenes with N50 length of $1400 \mathrm{bp}$ and total length of $44.5 \mathrm{Mb}$. Recently $\mathrm{Zhu}$ et al. generated an aluminum responsive transcriptome containing 39,815 contigs [58], whereas Logacheva et al. assembled a floral transcriptome containing 25,401 contigs [59] in F. tataricum. Thus, our high-throughput RNA-seq yielded considerably more genes. We speculated the gaps could result from the differences between salt stress and aluminum stress processes, or from the sequencing approaches. 
Among all transcriptome sequences, 36,688 (63.34\%) unigenes were annotated in public databases. Through GO classification, 20,834 (35.96\%) unigenes were categorized into 56 sub-categories, out of which 'metabolic process', 'binding', 'cellular process' and 'catalytic activity' were strongly represented. Meanwhile, KEGG and KOG classification were used as alternative approaches to map the unigenes to biological pathways. The results showed that metabolic pathways of 'Posttranslational modification, protein turnover, chaperones', 'Signal transduction mechanisms', 'Intracellular trafficking, secretion, and vesicular transport' in KOG and 'Signal transduction', 'Carbohydrate metabolism' and 'Translation' in KEGG were overrepresented. These results can supply with important cues for dissecting the molecular mechanisms of salt responses in F. tataricum.

Through comparison of gene expression patterns between control and salt treatment, we obtained 455 DEGs. This is relatively a smaller number compared with the DEGs numbers obtained in Arabidopsis [60,61], wheat [15], and rice [62]. We speculated that this gap could result from the fact that our RNA-seq data mainly reflected the early-stage gene expression profiles under salt stress $(24 \mathrm{~h}$ after salt treatment). Anyway, among these DEGs, the genes related to protein kinases, phosphatases, HSPs, ABC transporters, GSTs, abiotic-related transcription factors and circadian clock were dominant. Protein kinases and phosphatases act as key components in plant stress signaling [2]. Previous studies indicated that the mitogen-activated protein kinases (MAPK) pathways were tightly related to salt stress response [18,63-65]. Among our DEGs, c33984_g1 and c34545_g1, two homologs of MAPK kinase, were up-regulated after salinity treatment (Table 3), suggesting MAPK signaling pathways are also involved in buckwheat salt signaling. Meanwhile, four serine-threonine protein kinase encoding genes, namely $c 1037 \_1, c 25455 \_g 1, c 33021 \_g 2, c 25455 \_g 1$ and $c 22689 \_g 1$ showed significantly higher expression levels in salt treatment (Table 3), which was consistent with the observation in Spartina alterniflora salt stress transcriptome data [14]. In Arabidopsis thaliana, calcium signaling is elicited by salt stress, and calcium-dependent protein kinases (CDPKs) and CBL-interacting protein kinases (CIPKs) mediate the calcium signal and downstream responses $[1,4,66]$. It is worth noting that $c 38839 \_g 1, c 31052 \_g 1, c 60566 \_g 1$ (three putative CDPKs) and c49233_g1 (a putative CIPK) were up-regulated under salt stress (Table 3). Previous study showed that overexpression of a L-type lectin-domain-containing receptor kinase (AtLPK1) enhanced the salt-stress tolerance in Arabidopsis [67]. Coincidentally, our analysis showed that the expression of c10866_g1 (a homolog of AtLPK1) was increased by about 9 -fold in salt treatment than in control (Table 3). Besides, one putative protein phosphatase 2C (PP2C) gene (c38748_g1) and one tyrosine-protein phosphatase gene (c60922_g1) were significantly induced by salt stress (Table 3), suggesting protein phosphatases may play important roles in F. tataricum under stress.

In Arabidopsis and creeping bentgrass (Agrostis stolonifera L.), heat shock proteins (HSPs) function as protein chaperones to attenuate plant abiotic stress responses [68-70]. A lot of potential HSPs homologs identified by our study, such as c10992_g1, c22339_g1, c26417_g3, c29031_g1, $c 29386 \_g 1, c 29386 \_g 2, c 30033 \_g 1, c 31857 \_g 1, c 32499 \_g 1, c 32766 \_g 1, c 35678 \_g 1, c 38902 \_g 1$ and $c 54547 \_g 1$, were shown to accumulate more transcripts under salt stress (Table 3 ), suggesting the involvement of HSPs regulatory mechanisms. ATP-binding cassette $(\mathrm{ABC})$ transporters are also correlated with salt and other abiotic stress resistance [71,72]. Overexpression of AtABCG36 displayed higher fresh weight and stronger drought and salt resistance than wild type in Arabidopsis. Here, two ABC transporter B family members (c39646_g1 and c30623_g2) and two ABC transporter C family members (c38901_g1 and c33388_g1) were significantly up-regulated (Table 3), suggesting ABC transporters might share similar function in F. tataricum as well as in Arabidopsis. Glutathione S-transferases (GSTs) play vital roles in response to the oxidative stress including drought, salt and other stress [73-75]. Elevated expression levels of GSTs in tobacco and soybean were proven to enhance salt stress tolerance [74,75]. In our study, five putative GST encoding genes, namely c39155_g1,c39970_g1, c4684_g2, c59876_g1 and $c 60552 \_1$, showed significantly higher expression levels under salinity stress (Table 3 ), indicating GSTs might also impact the stress tolerance in F. tataricum. 
Brassinosteroid (BR) metabolism participates in regulating plant growth and abiotic stress resistance [56]. Genetic and biochemical evidences demonstrated that somatic embryogenesis receptor kinases (SERKs) interacted with brassinosteroid insensitive 1 (BRI1) and were indispensable for early steps in BR signaling [76]. In rice (Oryza sativa), interaction between 14-3-3 proteins and OsBZR1 was essential for maintaining robust BR responses [77]. Notably, the fact that $c 25693 \_g 1$ (putative SERK1) and c39300_g1 (predicted 14-3-3) were remarkably up-regulated under salt treated conditions (Table 3) indicated that BR signaling might also participate in salt stress signaling pathways in F. tataricum.

Using the PlantTFcat online tool, 3,107 out of 57,921 unigenes were predicted to encode for various putative transcription factors, which belong to 93 families. Interestingly, a great deal of abiotic stress responsive transcription factors, such as WD40-like, MYB, apetala2/ ethylene-responsive element-binding protein (AP2/EREBP), WRKY, bZIP and C2C2-Dof, were strongly dominant. Notably, many of them showed differentially expression patterns between control and salt treated conditions. In this study, four AP2/EREBP members (c21290_g1, c33171_g2,c44576_g1 and c50089_g1), one MYB member (c23951_g1), eight NAC members (c14111_g2,c15464_g1,c24596_g2, c26879_g1,c44171_g1, $c 44256 \_g 1, c 60319 \_g 1$ and c8889_g1), seven WRKY members (c10876_g1, c23849_g1, c25475_g1, $c 28962 \_g 2, c 45063 \_g 1, c 49337 \_g 1$ and $\left.c 49928 \_g 1\right)$ and three ZAT members $\left(c 38942 \_g 1, c 51237 \_g 1\right.$ and $\left.c 61192 \_1\right)$ were increased by more than 2 folds $\left(7.4>\log _{2}\right.$.fold changes $\left.>1.2\right)$ in salt treatment than control samples (Table 3). Accumulating evidence demonstrates that AP2/EREBP, MYB, NAC, WRKY and ZAT family members are closely associated with abiotic stress defense $[1,2,56,57,78]$. Overexpression of $A P 2$ /EREBP resulted in improved salt tolerance in rice and Arabidopsis [79]. In MYB family, OsMYB3R-2 and OsMYB48-1 overexpressors have been shown to harbor increased tolerance to cold, drought and salt stress in plant $[9,13]$. In soybean (Glycine max) and rice, NAC transcription factors were found to confer resistance to drought and salt stress $[7,11,80]$. Incorporation of wheat (Triticum aestivum) WRKY genes (TaWRKY2 and TaWRKY19) and soybean (GmWRKY13, GmWRKY21 and GmWRKY54) into Arabidopsis plants led to increased abiotic stress tolerance [10,12]. Mutation in ZAT7 abolished the salt tolerance, whereas elevated expression or RNA interference (RNAi) of ZAT10 gave rise to more tolerance to osmotic and salinity stress in Arabidopsis [8,81]. This indicates these transcription factors may confer the salinity adaption in F. tataricum, as well.

Circadian clock coordinates the plant growth and development in response to environment changes [82,83]. Many microarray and metabolite-profiling studies revealed that plant circadian clock controlled stress-related genes modulate the abiotic responses [84]. Mutation of Gigantea (GI) in Arabidopsis resulted in enhanced salt tolerance, which is due to the release of SOS2 kinase [85]. In addition, the rice osgi mutant exhibited increased osmotic stress and harbored higher abundance of OsDREB1E, OsAP37, OsAP59, OsLIP9, OsLEA3, OsRAB16A, and OsSalT [86]. Consistently, in our transcriptome data, c32034_g1 (predicted FtGIGANTEA in tartary buckwheat) was decreased by more than 2-fold under salt conditions (Table 3). This finding indicates that in FtGIGANTEA may also function in salt-stress adaption.

Taken together, we de novo generated and annotated a salt responsive transcriptome of F. tataricum. Genome-wide assay of the transcriptional differences between control and salt-treated conditions led to identification of a lot of key regulators in salt response mechanisms. Consequently, the present study could further improve our understanding of the salt tolerance mechanisms in F. tataricum. The transcriptome data could supply valuable molecular information for future plant breeding strategies. 
Table 3. Key regulators involved in salt stress responses in F. tataricum. CQ_0h and CQ_Na24h represent control and salt-treated samples respectively.

\begin{tabular}{|c|c|c|c|c|c|c|}
\hline Gene_ID & CQ_Na24_Readcount & CQ_0_Readcount & log2.Fold_Change & $p$-Value & $q$-Value & Annotation \\
\hline \multicolumn{7}{|c|}{ MAPK } \\
\hline c33984_\&1 & 149.2283698 & 47.67405994 & 1.6462 & $1.10 \mathrm{E}-14$ & $1.77 \mathrm{E}-12$ & Mitogen-activated protein kinase 9-like (Nelumbo nucifera) \\
\hline$c 34545 \_$\&1 & 59.3142507 & 5.238326231 & 3.5012 & $1.99 \mathrm{E}-13$ & $2.75 \mathrm{E}-11$ & Mitogen-activated protein kinase 2-like (Citrus sinensis) \\
\hline \multicolumn{7}{|c|}{ Serine-threonine protein kinase } \\
\hline c1037_g1 & 36.96338405 & 1.587371585 & 4.5414 & $3.94 \mathrm{E}-10$ & $3.99 \mathrm{E}-08$ & probable serine-threonine protein kinase (Beta vulgaris subsp. vulgaris) \\
\hline c25455_g1 & 32.83888317 & 1.957758288 & 4.0681 & $9.25 \mathrm{E}-09$ & $7.77 \mathrm{E}-07$ & serine-threonine protein kinase (Ricinus communis) \\
\hline$c 33021 \_2$ & 18.93342307 & 1.005335337 & 4.2352 & $1.04 \mathrm{E}-05$ & 0.0005562 & serine-threonine protein kinase (Ricinus communis) \\
\hline$c 25455 \_11$ & 32.83888317 & 1.957758288 & 4.0681 & $9.25 \mathrm{E}-09$ & $7.77 \mathrm{E}-07$ & serine-threonine protein kinase (Ricinus communis) \\
\hline$c 22689 \_1$ & 45.015981 & 15.55624154 & 1.5329 & $5.30 \mathrm{E}-05$ & 0.0024396 & serine/threonine protein kinase (Eucalyptus grandis) \\
\hline \multicolumn{7}{|c|}{ CDPKs } \\
\hline c38839_81 & 35.23502178 & 8.042682698 & 2.1313 & $9.55 \mathrm{E}-06$ & 0.00051234 & Calcium-dependent protein kinase 19 (Theobroma cacao) \\
\hline$c 31052 \_11$ & 232.8033275 & 105.0294992 & 1.1483 & $8.13 \mathrm{E}-14$ & $1.17 \mathrm{E}-11$ & Calcium-dependent protein kinase 26 isoform X1 (Vitis vinifera) \\
\hline$c 60566-81$ & 41.9127851 & 14.39216904 & 1.5421 & $8.99 \mathrm{E}-05$ & 0.0039211 & Calcium-dependent protein kinase 11 (Beta vulgaris subsp. vulgaris) \\
\hline \multicolumn{7}{|c|}{ CIPKs } \\
\hline c49233_g1 & 703.207759 & 280.4356467 & 1.3263 & $3.54 \mathrm{E}-47$ & $2.54 \mathrm{E}-44$ & CBL-interacting serine/threonine-protein kinase 4-like (Beta vulgaris subsp. vulgaris) \\
\hline \multicolumn{7}{|r|}{ e } \\
\hline c10866_81 & 25.21837679 & 2.751444081 & 3.1962 & $3.82 \mathrm{E}-06$ & 0.00022042 & kinase, putative (Ricinus communis) \\
\hline \multicolumn{7}{|c|}{ PP2C } \\
\hline c38748_g1 & 173.1111939 & 63.54777579 & 1.4458 & $2.75 \mathrm{E}-14$ & $4.10 \mathrm{E}-12$ & protein phosphatase 2C 6 (Morus notabilis) \\
\hline \multicolumn{7}{|c|}{ PTPase } \\
\hline c60922_s1 & 38.06325095 & 6.137836796 & 2.6326 & $2.23 \mathrm{E}-07$ & $1.57 \mathrm{E}-05$ & tyrosine-protein phosphatase (Beta vulgaris subsp. vulgaris) \\
\hline \multicolumn{7}{|c|}{ HSPs } \\
\hline c10992_81 & 595.8528933 & 171.2244817 & 1.7991 & $2.80 \mathrm{E}-60$ & $2.80 \mathrm{E}-57$ & heat shock protein 70 (Ageratina adenophora) \\
\hline c22339_g1 & 128.9593941 & 50.9017155 & 1.3411 & $4.77 \mathrm{E}-10$ & $4.76 \mathrm{E}-08$ & heat shock protein, putative (Ricinus communis) \\
\hline$c 26417 \_3$ & 280.7410263 & 129.0003975 & 1.1219 & $7.62 \mathrm{E}-16$ & $1.36 \mathrm{E}-13$ & Heat shock cognate 70 kDa protein (Glycine soja) \\
\hline$c 29031 \_81$ & 123.4207786 & 52.065788 & 1.2452 & $7.83 \mathrm{E}-09$ & $6.64 \mathrm{E}-07$ & hypothetical protein B456_013G139100 (Gossypium raimondii) \\
\hline$c 29386 \_$_1 & 1492.912194 & 346.1528304 & 2.1086 & $1.92 \mathrm{E}-180$ & $1.06 \mathrm{E}-176$ & heat shock protein 83 (Beta vulgaris subsp. vulgaris) \\
\hline$c 29386 \_g^{2}$ & 864.9274743 & 402.3986969 & 1.104 & $2.04 \mathrm{E}-44$ & $1.37 \mathrm{E}-41$ & heat shock protein 90-2 (Populus euphratica) \\
\hline c30033_g1 & 222.2909568 & 78.20450677 & 1.5071 & $6.84 \mathrm{E}-19$ & $1.60 \mathrm{E}-16$ & heat shock protein 70 (Gossypium hirsutum) \\
\hline c31857_g1 & 196.9940181 & 78.25741915 & 1.3319 & $1.90 \mathrm{E}-14$ & $2.88 \mathrm{E}-12$ & class I heat shock protein-like (Nelumbo nucifera) \\
\hline$c 32499 \_-g 1$ & 674.21841 & 200.4321188 & 1.7501 & $1.27 \mathrm{E}-65$ & $1.50 \mathrm{E}-62$ & heat shock protein 2 (Sesamum indicum) \\
\hline c32766_\&1 & 93.5279675 & 46.50998745 & 1.0079 & $1.78 \mathrm{E}-05$ & 0.00091511 & heat shock 70 kDa protein 14-like (Vitis vinifera) \\
\hline$c 35678-g 1$ & 57.62516939 & 7.354821678 & 2.9699 & $1.37 \mathrm{E}-11$ & $1.59 \mathrm{E}-09$ & class II heat shock protein-like (Populus euphratica) \\
\hline$c 38902 \_81$ & 275.9880301 & 87.94038582 & 1.65 & $6.68 \mathrm{E}-26$ & $2.40 \mathrm{E}-23$ & class I heat shock protein (Vitis vinifera) \\
\hline c54547_g1 & 852.1611621 & 267.4721121 & 1.6717 & $1.06 \mathrm{E}-77$ & $1.40 \mathrm{E}-74$ & heat shock protein (Solanum lycopersicum) \\
\hline \multicolumn{7}{|c|}{ ABC Transporter } \\
\hline c39646_g1 & 110.6151854 & 44.97552825 & 1.2983 & $1.77 \mathrm{E}-08$ & $1.44 \mathrm{E}-06$ & ABC transporter B family member 25 (Vitis vinifera) \\
\hline$c 30623 \_2$ & 110.5759045 & 22.06446503 & 2.3252 & $1.56 \mathrm{E}-16$ & $2.92 \mathrm{E}-14$ & ABC transporter B family member 11-like (Beta vulgaris subsp. vulgaris) \\
\hline$c 38901-81$ & 526.9933692 & 238.2115626 & 1.1455 & $3.36 \mathrm{E}-29$ & $1.32 \mathrm{E}-26$ & $\mathrm{ABC}$ transporter $\mathrm{C}$ family member 4 (Gossypium arboreum) \\
\hline c33388_81 & 55.58255943 & 10.10626576 & 2.4594 & $1.58 \mathrm{E}-09$ & $1.45 \mathrm{E}-07$ & ABC transporter $\mathrm{C}$ family member 3-like (Pyrus $\times$ bretschneideri) \\
\hline
\end{tabular}


Table 3. Cont.

\begin{tabular}{|c|c|c|c|c|c|c|}
\hline Gene_ID & CQ_Na24_Readcount & CQ_0_Readcount & log2.Fold_Change & $p$-Value & $q$-Value & Annotation \\
\hline \multicolumn{7}{|c|}{ GST } \\
\hline c39155_81 & 250.1804389 & 40.74253735 & 2.6184 & $5.03 \mathrm{E}-40$ & $2.97 \mathrm{E}-37$ & glutathione S-transferase (Eucalyptus grandis) \\
\hline c39970_81 & 45.17310484 & 12.32858598 & 1.8735 & $3.69 \mathrm{E}-06$ & 0.00021425 & glutathione transferase (Populus trichocarpa) \\
\hline$c 4684-g^{2}$ & 107.5119895 & 14.60381858 & 2.8801 & $8.78 \mathrm{E}-20$ & $2.30 \mathrm{E}-17$ & glutathione S-transferase (Eucalyptus grandis) \\
\hline$c 59876 \_-81$ & 188.4307686 & 61.59001751 & 1.6133 & $1.09 \mathrm{E}-17$ & $2.21 \mathrm{E}-15$ & glutathione S-transferase (Citrus sinensis) \\
\hline$c 60552 \_81$ & 79.93675509 & 7.19608452 & 3.4736 & $1.79 \mathrm{E}-17$ & $3.55 \mathrm{E}-15$ & glutathione S-transferase U17-like (Citrus sinensis) \\
\hline \multicolumn{7}{|c|}{ SERK1 } \\
\hline c25693_81 & 21.29028072 & 0.634948634 & 5.0674 & $1.37 \mathrm{E}-06$ & $8.58 \mathrm{E}-05$ & somatic embryogenesis receptor kinase 1-like (Zea mays) \\
\hline \multicolumn{7}{|c|}{$14-3-3$} \\
\hline c39300_81 & 82.49001753 & 39.10225338 & 1.077 & $2.27 \mathrm{E}-05$ & 0.0011344 & 14-3-3 protein, putative (Ricinus communis) \\
\hline \multicolumn{7}{|c|}{ AP2/EREBP } \\
\hline$c 21290 \_81$ & 39.55592746 & 8.201419857 & 2.2699 & $1.13 \mathrm{E}-06$ & 7.13E-05 & ethylene-responsive transcription factor (Sesamum indicum) \\
\hline$c 33171 \_2$ & 52.40080161 & 18.94263425 & 1.468 & $2.32 \mathrm{E}-05$ & 0.0011555 & AP2-like ethylene-responsive transcription factor AIL5 (Eucalyptus grandis) \\
\hline$c 44576 \_1$ & 67.79893822 & 28.67851331 & 1.2413 & $1.97 \mathrm{E}-05$ & 0.00099714 & ethylene-responsive transcription factor ABR1-like (Populus euphratica) \\
\hline$c 50089 \_1$ & 84.41478461 & 14.23343188 & 2.5682 & $2.66 \mathrm{E}-14$ & $3.99 \mathrm{E}-12$ & putative ethylene response factor 5 (Vitis aestivalis) \\
\hline \multicolumn{7}{|c|}{ MYB } \\
\hline c23951_s1 & 55.8182452 & 19.84214482 & 1.4922 & $9.98 \mathrm{E}-06$ & 0.00053404 & transcription factor MYB108 (Beta vulgaris subsp. vulgaris) \\
\hline \multicolumn{7}{|c|}{ NAC } \\
\hline$c 14111 \_g^{2}$ & 46.90146711 & 6.190749182 & 2.9214 & $1.42 \mathrm{E}-09$ & $1.32 \mathrm{E}-07$ & NAC domain-containing protein (Boehmeria nivea) \\
\hline$c 15464 \_1$ & 48.47270554 & 0.846598179 & 5.8394 & $2.27 \mathrm{E}-13$ & 3.13E -11 & NAC transcription factor (Fagus sylvatica) \\
\hline$c 24596 \_g^{2}$ & 89.3249047 & 35.76877305 & 1.3204 & $2.99 \mathrm{E}-07$ & $2.07 \mathrm{E}-05$ & NAC domain-containing protein 2-like (Tarenaya hassleriana) \\
\hline$c 26879 \_1$ & 85.8288992 & 30.53044682 & 1.4912 & $4.36 \mathrm{E}-08$ & $3.41 \mathrm{E}-06$ & NAC domain-containing protein 78 (Vitis vinifera) \\
\hline$c 44171_{-81}$ & 65.59920442 & 29.63093626 & 1.1466 & $7.51 \mathrm{E}-05$ & 0.0033343 & NAC domain protein (Medicago truncatula) \\
\hline$c 44256 \_81$ & 232.5040066 & 87.56999912 & 1.4087 & $4.75 \mathrm{E}-18$ & $1.01 \mathrm{E}-15$ & NAC transcription factor (Fagus sylvatica) \\
\hline$c 60319 \_1$ & 54.52197349 & 8.99510565 & 2.5996 & $7.35 \mathrm{E}-10$ & $7.10 \mathrm{E}-08$ & NAC domain-containing protein (Boehmeria nivea) \\
\hline$c 8889-g 1$ & 97.37750165 & 31.27122023 & 1.6388 & $4.90 \mathrm{E}-10$ & $4.87 \mathrm{E}-08$ & NAC domain-containing protein 1 (Salvia miltiorrhiza) \\
\hline \multicolumn{7}{|c|}{ WRKY } \\
\hline c10876_81 & 37.67044134 & 2.222320219 & 4.0833 & $7.35 \mathrm{E}-10$ & $7.10 \mathrm{E}-08$ & WRKY transcription factor 31 (Nicotiana sylvestris) \\
\hline$c 23849 \_1$ & 215.1811029 & 69.89726214 & 1.6222 & $4.05 \mathrm{E}-20$ & $1.10 \mathrm{E}-17$ & WRKY protein (Rheum australe) \\
\hline$c 25475 \_-81$ & 64.77430424 & 13.3868337 & 2.2746 & $4.47 \mathrm{E}-10$ & $4.47 \mathrm{E}-08$ & WRKY4 transcription factor (Vitis aestivalis) \\
\hline$c 28962-8^{2}$ & 179.0819 & 55.55800548 & 1.6886 & $7.33 \mathrm{E}-18$ & $1.52 \mathrm{E}-15$ & WRKY transcription factor 33 (Glycine max) \\
\hline$c 45063 \_g 1$ & 30.24633976 & 3.439305101 & 3.1366 & $5.17 \mathrm{E}-07$ & $3.43 \mathrm{E}-05$ & WRKY transcription factor 75 (Nelumbo nucifera) \\
\hline$c 49337 \_1$ & 37.04194597 & 4.762114756 & 2.9595 & $6.23 \mathrm{E}-08$ & $4.76 \mathrm{E}-06$ & WRKY transcription factor 70-like (Citrus sinensis) \\
\hline$c 49928 \_1$ & 18.1085229 & 0.105824772 & 7.4188 & $1.57 \mathrm{E}-05$ & 0.00081139 & WRKY transcription factor 73 (Gossypium hirsutum) \\
\hline \multicolumn{7}{|r|}{ 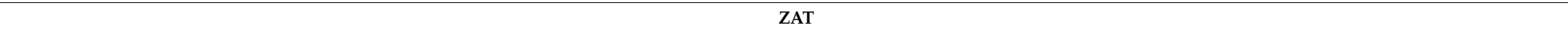 } \\
\hline & 96.94541108 & 21.85281549 & 2.1494 & $1.57 \mathrm{E}-13$ & $2.22 \mathrm{E}-11$ & zinc finger protein ZAT10 (Beta vulgaris subsp. vulgaris) \\
\hline$c 51237-g 1$ & 19.79760421 & 0.423299089 & 5.5475 & $2.80 \mathrm{E}-06$ & 0.00016544 & zinc finger protein ZAT11-like (Solanum tuberosum) \\
\hline$c 61192 \_$_1 & 52.32223969 & 6.08492441 & 3.1041 & $4.95 \mathrm{E}-11$ & $5.54 \mathrm{E}-09$ & zinc finger protein ZAT10 (Populus euphratica) \\
\hline \multicolumn{7}{|r|}{ protein GIGANTFA (Rota zularaic subsn zulogric) } \\
\hline c32034_81 & 202.2969478 & 445.575204 & -1.1392 & $1.05 \mathrm{E}-19$ & $2.73 \mathrm{E}-17$ & protein GIGANTEA (Beta vulgaris subsp. vulgaris) \\
\hline
\end{tabular}

MAPK: mitogen-activated protein kinase; CDPKs: calcium-dependent protein kinases; CIPKs: CBL-interacting serine/threonine--protein kinases; LPK: L-type lectin-domain-containing receptor kinase; PP2C: protein phosphatase 2C; PTPase: tyrosine-protein phosphatase; HSPs: heat shock proteins; ABC Transporter: ATP-binding cassette Transporter; GST: glutathione NAC: NAC transcription factor; WRKY: WRKY transcription factor; ZAT: zinc finger protein ZAT. 
Supplementary Materials: The following are available online at www.mdpi.com/2073-4425/8/10/255/s1. Figure S1: Numbers of up- and down-regulated genes between control and salt treatment in Fagopyrum. tataricum. Table S1: Gene ontology of the F. tataricum transcriptome at level2. Table S2: Statistics of euKaryotic Orthologous Groups (KOG) function classification of the F. tataricum transcriptome. Table S3: Enrichment of the F. tataricum transcriptome on Kyoto Encyclopedia of Genes and Genomes (KEGG) pathways at Hierarchy2. Table S4: Identification of the transcription factor families in F. tataricum. Table S5: Gene ontology of the differentially expressed genes (DEGs). Table S6: Enrichment of the DEGs on KEGG pathways. Table S7: Statistics of the frequency for different simple sequence repeats (SSRs) in F. tataricum. Table S8: The primers used for real-time PCR in this study.

Acknowledgments: This work was supported by the National Natural Science Foundation of China (31701493), the China Agriculture Research System (CARS-08-02A), and the Chengdu University Research Fund (2080517045; 2081916061; 2080516031). We would like to thank Qingtian Li (Department of Botany and Plant Sciences, University of California, Riverside, CA, USA) and Lixiang Wang (College of Plant Science and Technology, Huazhong Agriculture University, China) for critical reading on the manuscript.

Author Contributions: Q.W. and G.Z. conceived and designed the experiments. Q.W. and X.B. performed most of the experiments. Q.W., X.B., and G.Z. wrote the manuscript. W.Z., D.X. and Y.W. conducted salt treatment, physiological analysis, and prepared the RNA-seq materials. Q.W., X.B., J.Y. and L.Z. performed data analysis. All authors have read and approved the manuscript.

Conflicts of Interest: The authors declare no conflict of interest.

\section{References}

1. Zhu, J.K. Salt and drought stress signal transduction in plants. Annu. Rev. Plant Biol. 2002, 53, $247-273$. [CrossRef] [PubMed]

2. Munns, R.; Tester, M. Mechanisms of salinity tolerance. Annu. Rev. Plant Biol. 2008, 59, 651-681. [CrossRef] [PubMed]

3. Hasegawa, P.M.; Bressan, R.A.; Zhu, J.K.; Bohnert, H.J. Plant Cellular and Molecular Responses to High Salinity. Annu. Rev. Plant Physiol. Plant Mol. Biol. 2000, 51, 463-499. [CrossRef] [PubMed]

4. Weinl, S.; Kudla, J. The CBL-CIPK Ca ${ }^{2+}$-decoding signaling network: function and perspectives. New Phytol. 2009, 184, 517-528. [CrossRef] [PubMed]

5. Apel, K.; Hirt, H. Reactive oxygen species: Metabolism, oxidative stress, and signal transduction. Annu. Rev. Plant Biol. 2004, 55, 373-399. [CrossRef] [PubMed]

6. Cabello, J.V.; Lodeyro, A.F.; Zurbriggen, M.D. Novel perspectives for the engineering of abiotic stress tolerance in plants. Curr. Opin. Biotechnol. 2014, 26, 62-70. [CrossRef] [PubMed]

7. Hu, H.; Dai, M.; Yao, J.; Xiao, B.; Li, X.; Zhang, Q.; Xiong, L. Overexpressing a NAM, ATAF, and CUC (NAC) transcription factor enhances drought resistance and salt tolerance in rice. Proc. Natl. Acad. Sci. USA 2006, 103, 12987-12992. [CrossRef] [PubMed]

8. Ciftci-Yilmaz, S.; Morsy, M.R.; Song, L.; Coutu, A.; Krizek, B.A.; Lewis, M.W.; Warren, D.; Cushman, J.; Connolly, E.L.; Mittler, R. The EAR-motif of the Cys2/His2-type zinc finger protein Zat7 plays a key role in the defense response of Arabidopsis to salinity stress. J. Biol. Chem. 2007, 282, 9260-9268. [CrossRef] [PubMed]

9. Dai, X.; Xu, Y.; Ma, Q.; Xu, W.; Wang, T.; Xue, Y.; Chong, K. Overexpression of an R1R2R3 MYB gene, OsMYB3R-2, increases tolerance to freezing, drought, and salt stress in transgenic Arabidopsis. Plant Physiol. 2007, 143, 1739-1751. [CrossRef] [PubMed]

10. Zhou, Q.Y.; Tian, A.G.; Zou, H.F.; Xie, Z.M.; Lei, G.; Huang, J.; Wang, C.M.; Wang, H.W.; Zhang, J.S.; Chen, S.Y. Soybean WRKY-type transcription factor genes, GmWRKY13, GmWRKY21, and GmWRKY54, confer differential tolerance to abiotic stresses in transgenic Arabidopsis plants. Plant Biotechnol. J. 2008, 6, 486-503. [CrossRef] [PubMed]

11. Hao, Y.J.; Wei, W.; Song, Q.X.; Chen, H.W.; Zhang, Y.Q.; Wang, F.; Zou, H.F.; Lei, G.; Tian, A.G.; Zhang, W.K.; et al. Soybean NAC transcription factors promote abiotic stress tolerance and lateral root formation in transgenic plants. Plant. J. 2011, 68, 302-313. [CrossRef] [PubMed]

12. Niu, C.F.; Wei, W.; Zhou, Q.Y.; Tian, A.G.; Hao, Y.J.; Zhang, W.K.; Ma, B.; Lin, Q.; Zhang, Z.B.; Zhang, J.S.; Chen, S.Y. Wheat WRKY genes TaWRKY2 and TaWRKY19 regulate abiotic stress tolerance in transgenic Arabidopsis plants. Plant Cell. Environ. 2012, 35, 1156-1170. [CrossRef] [PubMed]

13. Xiong, H.; Li, J.; Liu, P.; Duan, J.; Zhao, Y.; Guo, X.; Li, Y.; Zhang, H.; Ali, J.; Li, Z. Overexpression of OsMYB48-1, a novel MYB-related transcription factor, enhances drought and salinity tolerance in rice. PLoS ONE 2014, 9, e92913. [CrossRef] [PubMed] 
14. Bedre, R.; Mangu, V.R.; Srivastava, S.; Sanchez, L.E.; Baisakh, N. Transcriptome analysis of smooth cordgrass (Spartina alterniflora Loisel), a monocot halophyte, reveals candidate genes involved in its adaptation to salinity. BMC Genomics 2016, 17, 657. [CrossRef] [PubMed]

15. Goyal, E.; Amit, S.K.; Singh, R.S.; Mahato, A.K.; Chand, S.; Kanika, K. Transcriptome profiling of the salt-stress response in Triticum aestivum cv. Kharchia Local. Sci. Rep. 2016, 6, 27752. [CrossRef] [PubMed]

16. Hill, C.B.; Cassin, A.; Keeble-Gagnere, G.; Doblin, M.S.; Bacic, A.; Roessner, U. De novo transcriptome assembly and analysis of differentially expressed genes of two barley genotypes reveal root-zone-specific responses to salt exposure. Sci. Rep. 2016, 6, 31558. [CrossRef] [PubMed]

17. Jin, H.; Dong, D.; Yang, Q.; Zhu, D. Salt-Responsive Transcriptome Profiling of Suaeda glauca via RNA Sequencing. PLoS ONE 2016, 11, e0150504. [CrossRef] [PubMed]

18. Shankar, R.; Bhattacharjee, A.; Jain, M. Transcriptome analysis in different rice cultivars provides novel insights into desiccation and salinity stress responses. Sci. Rep. 2016, 6, 23719. [CrossRef] [PubMed]

19. Mallikarjuna, G.; Mallikarjuna, K.; Reddy, M.K.; Kaul, T. Expression of OsDREB2A transcription factor confers enhanced dehydration and salt stress tolerance in rice (Oryza sativa L.). Biotechnol. Lett. 2011, 33, 1689-1697. [CrossRef] [PubMed]

20. Wang, C.; Lu, G.; Hao, Y.; Guo, H.; Guo, Y.; Zhao, J.; Cheng, H. ABP9, a maize bZIP transcription factor, enhances tolerance to salt and drought in transgenic cotton. Planta 2017, 246, 453-469. [CrossRef] [PubMed]

21. Zhao, C.; Zhu, J.K. The broad roles of CBF genes: From development to abiotic stress. Plant Signal. Behav. 2016, 11, e1215794. [CrossRef] [PubMed]

22. Rahman, H.; Ramanathan, V.; Nallathambi, J.; Duraialagaraja, S.; Muthurajan, R. Over-expression of a NAC 67 transcription factor from finger millet (Eleusine coracana L.) confers tolerance against salinity and drought stress in rice. BMC Biotechnol. 2016, 16, 35. [CrossRef] [PubMed]

23. Buermans, H.P.; den Dunnen, J.T. Next generation sequencing technology: Advances and applications. Biochim. Biophys. Acta 2014, 1842, 1932-1941. [CrossRef] [PubMed]

24. Wang, Z.; Gerstein, M.; Snyder, M. RNA-Seq: A revolutionary tool for transcriptomics. Nat. Rev. Genet. 2009, 10, 57-63. [CrossRef] [PubMed]

25. Li, Y.Q.; Shi, T.L.; Zhang, Z.W. Development of microsatellite markers from Tartary buckwheat. Biotechnol. Lett. 2007, 29, 823-827. [CrossRef] [PubMed]

26. Li, C.; Kobayashi, K.; Yoshida, Y.; Ohsawa, R. Genetic analyses of agronomic traits in Tartary buckwheat (Fagopyrum tataricum (L.) Gaertn.). Breed. Sci. 2012, 62, 303-309. [CrossRef] [PubMed]

27. Chen, L.H.; Zhang, B.; Xu, Z.Q. Salt tolerance conferred by overexpression of Arabidopsis vacuolar $\mathrm{Na}^{+} / \mathrm{H}^{+}$ antiporter gene AtNHX1 in common buckwheat (Fagopyrum esculentum). Transgenic Res. 2008, 17, 121-132. [CrossRef] [PubMed]

28. Zhang, L.; Li, X.; Ma, B.; Gao, Q.; Du, H.; Han, Y.; Li, Y.; Cao, Y.; Qi, M.; Zhu, Y.; et al. The Tartary Buckwheat Genome Provides Insights into Rutin Biosynthesis and Abiotic Stress Tolerance. Mol. Plant 2017, 10, 1224-1237. [CrossRef] [PubMed]

29. Li, Z.; Baldwin, C.M.; Hu, Q.; Liu, H.; Luo, H. Heterologous expression of Arabidopsis $\mathrm{H}^{+}$-pyrophosphatase enhances salt tolerance in transgenic creeping bentgrass (Agrostis stolonifera L.). Plant Cell Environ. 2010, 33, 272-289. [CrossRef] [PubMed]

30. Ishitani, M.; Xiong, L.; Lee, H.; Stevenson, B.; Zhu, J.K. HOS1, a genetic locus involved in cold-responsive gene expression in Arabidopsis. Plant Cell 1998, 10, 1151-1161. [CrossRef] [PubMed]

31. Shan, H.; Liang, Z.; Shao, M. Changes of anti-oxidative enzymes and MDA content under soil water deficits among 10 wheat (Triticum aestivum L.) genotypes at maturation stage. Colloids Surf. B. Biointerfaces 2005, 45, 7-13.

32. Grabherr, M.G.; Haas, B.J.; Yassour, M.; Levin, J.Z.; Thompson, D.A.; Amit, I.; Adiconis, X.; Fan, L.; Raychowdhury, R.; Zeng, Q.; et al. Full-length transcriptome assembly from RNA-Seq data without a reference genome. Nat Biotechnol 2011, 29, 644-652. [CrossRef] [PubMed]

33. Pruitt, K.D.; Tatusova, T.; Maglott, D.R. NCBI reference sequences (RefSeq): A curated non-redundant sequence database of genomes, transcripts and proteins. Nucleic Acids Res. 2007, 35, D61-D65. [CrossRef] [PubMed]

34. Kuimelis, R.G.; Livak, K.J.; Mullah, B.; Andrus, A. Structural analogues of TaqMan probes for real-time quantitative PCR. Nucleic Acids Symp. Ser. 1997, 37, 255-256. 
35. Altschul, S.F.; Gish, W.; Miller, W.; Myers, E.W.; Lipman, D.J. Basic Local Alignment Search Tool. J. Mol. Biol. 1990, 215, 403-410. [CrossRef]

36. Iseli, C.; Jongeneel, C.V.; Bucher, P. ESTScan: A program for detecting, evaluating, and reconstructing potential coding regions in EST sequences. Proc. Int. Conf. Intell. Syst. Mol. Biol. 1999, 138-148.

37. Livak, K.J.; Schmittgen, T.D. Analysis of relative gene expression data using real-time quantitative PCR and the $2^{\wedge}(-\Delta \Delta \mathrm{CT})$ Method. Methods 2001, 25, 402-408. [CrossRef] [PubMed]

38. Heid, C.A.; Stevens, J.; Livak, K.J.; Williams, P.M. Real time quantitative PCR. Genome Res. 1996, 6, $986-994$. [CrossRef] [PubMed]

39. Moriya, Y.; Itoh, M.; Okuda, S.; Yoshizawa, A.C.; Kanehisa, M. KAAS: An automatic genome annotation and pathway reconstruction server. Nucleic Acids Res. 2007, 35, W182-W185. [CrossRef] [PubMed]

40. Dai, X.; Sinharoy, S.; Udvardi, M.; Zhao, P.X. PlantTFcat: An online plant transcription factor and transcriptional regulator categorization and analysis tool. BMC Bioinformatics 2013, 14, 321. [CrossRef] [PubMed]

41. Gotz, S.; Garcia-Gomez, J.M.; Terol, J.; Williams, T.D.; Nagaraj, S.H.; Nueda, M.J.; Robles, M.; Talon, M.; Dopazo, J.; Conesa, A. High-throughput functional annotation and data mining with the Blast2GO suite. Nucleic Acids Res. 2008, 36, 3420-3435. [CrossRef] [PubMed]

42. Finn, R.D.; Tate, J.; Mistry, J.; Coggill, P.C.; Sammut, S.J.; Hotz, H.R.; Ceric, G.; Forslund, K.; Eddy, S.R.; Sonnhammer, E.L.; et al. The Pfam protein families database. Nucleic Acids Res. 2008, 36, D281-D288. [CrossRef] [PubMed]

43. Finn, R.D.; Clements, J.; Eddy, S.R. HMMER web server: Interactive sequence similarity searching. Nucleic Acids Res. 2011, 39, W29-W37. [CrossRef] [PubMed]

44. Langmead, B.; Salzberg, S.L. Fast gapped-read alignment with Bowtie 2. Nat. Methods 2012, 9, 357-359. [CrossRef] [PubMed]

45. Li, B.; Dewey, C.N. RSEM: Accurate transcript quantification from RNA-Seq data with or without a reference genome. BMC Bioinform. 2011, 12, 323. [CrossRef] [PubMed]

46. Trapnell, C.; Williams, B.A.; Pertea, G.; Mortazavi, A.; Kwan, G.; van Baren, M.J.; Salzberg, S.L.; Wold, B.J.; Pachter, L. Transcript assembly and quantification by RNA-Seq reveals unannotated transcripts and isoform switching during cell differentiation. Nat. Biotechnol. 2010, 28, 511-515. [CrossRef] [PubMed]

47. Robinson, M.D.; Oshlack, A. A scaling normalization method for differential expression analysis of RNA-Seq data. Genome Biol. 2010, 11, R25. [CrossRef] [PubMed]

48. Wang, L.; Feng, Z.; Wang, X.; Wang, X.; Zhang, X. DEGseq: An R package for identifying differentially expressed genes from RNA-Seq data. Bioinformatics 2010, 26, 136-138. [CrossRef] [PubMed]

49. Young, M.D.; Wakefield, M.J.; Smyth, G.K.; Oshlack, A. Gene ontology analysis for RNA-Seq: Accounting for selection bias. Genome Biol. 2010, 11, R14. [CrossRef] [PubMed]

50. Mao, X.; Cai, T.; Olyarchuk, J.G.; Wei, L. Automated genome annotation and pathway identification using the KEGG Orthology (KO) as a controlled vocabulary. Bioinformatics 2005, 21, 3787-3793. [CrossRef] [PubMed]

51. Thiel, T.; Michalek, W.; Varshney, R.K.; Graner, A. Exploiting EST databases for the development and characterization of gene-derived SSR-markers in barley (Hordeum vulgare L.). Theor. Appl. Genet. 2003, 106, 411-422. [CrossRef] [PubMed]

52. Fang, Z.; Zhang, X.; Gao, J.; Wang, P.; Xu, X.; Liu, Z.; Shen, S.; Feng, B. A Buckwheat (Fagopyrum esculentum) DRE-Binding Transcription Factor Gene, FeDREB1, Enhances Freezing and Drought Tolerance of Transgenic Arabidopsis. Plant Mol. Biol. Report. 2015, 33, 1510-1525. [CrossRef]

53. Nakashima, K.; Ito, Y.; Yamaguchi-Shinozaki, K. Transcriptional regulatory networks in response to abiotic stresses in Arabidopsis and grasses. Plant Physiol. 2009, 149, 88-95. [CrossRef] [PubMed]

54. Yang, Z.; Chen, Z.; Peng, Z.; Yu, Y.; Liao, M.; Wei, S. Development of a high-density linkage map and mapping of the three-pistil gene (Pis1) in wheat using GBS markers. BMC Genom. 2017, 18, 567. [CrossRef] [PubMed]

55. Li, S.; Fan, C.; Li, Y.; Zhang, J.; Sun, J.; Chen, Y.; Tian, C.; Su, X.; Lu, M.; Liang, C.; et al. Effects of drought and salt-stresses on gene expression in Caragana korshinskii seedlings revealed by RNA-seq. BMC Genom. 2016, 17, 200. [CrossRef] [PubMed]

56. Roy, S.J.; Negrao, S.; Tester, M. Salt resistant crop plants. Curr. Opin. Biotechnol. 2014, 26, 115-124. [CrossRef] [PubMed]

57. Zhu, J.K. Abiotic Stress Signaling and Responses in Plants. Cell 2016, 167, 313-324. [CrossRef] [PubMed] 
58. Zhu, H.; Wang, H.; Zhu, Y.; Zou, J.; Zhao, F.J.; Huang, C.F. Genome-wide transcriptomic and phylogenetic analyses reveal distinct aluminum-tolerance mechanisms in the aluminum-accumulating species buckwheat (Fagopyrum tataricum). BMC Plant Biol. 2015, 15, 16. [CrossRef] [PubMed]

59. Logacheva, M.D.; Kasianov, A.S.; Vinogradov, D.V.; Samigullin, T.H.; Gelfand, M.S.; Makeev, V.J.; Penin, A.A. De novo sequencing and characterization of floral transcriptome in two species of buckwheat (Fagopyrum). BMC Genom. 2011, 12, 30. [CrossRef] [PubMed]

60. Taji, T.; Seki, M.; Satou, M.; Sakurai, T.; Kobayashi, M.; Ishiyama, K.; Narusaka, Y.; Narusaka, M.; Zhu, J.K.; Shinozaki, K. Comparative genomics in salt tolerance between Arabidopsis and Arabidopsis-related halophyte salt cress using Arabidopsis microarray. Plant Physiol. 2004, 135, 1697-1709. [CrossRef] [PubMed]

61. Gong, Q.; Li, P.; Ma, S.; Indu Rupassara, S.; Bohnert, H.J. Salinity stress adaptation competence in the extremophile Thellungiella halophila in comparison with its relative Arabidopsis thaliana. Plant J. 2005, 44, 826-839. [CrossRef] [PubMed]

62. Do Amaral, M.N.; Arge, L.W.; Benitez, L.C.; Danielowski, R.; Silveira, S.F.; Farias Dda, R.; de Oliveira, A.C.; da Maia, L.C.; Braga, E.J. Comparative transcriptomics of rice plants under cold, iron, and salt stresses. Funct. Integr. Genom. 2016, 16, 567-579. [CrossRef] [PubMed]

63. Nakagami, H.; Pitzschke, A.; Hirt, H. Emerging MAP kinase pathways in plant stress signalling. Trends Plant Sci. 2005, 10, 339-346. [CrossRef] [PubMed]

64. Sinha, A.K.; Jaggi, M.; Raghuram, B.; Tuteja, N. Mitogen-activated protein kinase signaling in plants under abiotic stress. Plant Signal Behav. 2011, 6, 196-203. [CrossRef] [PubMed]

65. Kiegerl, S.; Cardinale, F.; Siligan, C.; Gross, A.; Baudouin, E.; Liwosz, A.; Eklof, S.; Till, S.; Bogre, L.; Hirt, H.; et al. SIMKK, a mitogen-activated protein kinase (MAPK) kinase, is a specific activator of the salt stress-induced MAPK, SIMK. Plant Cell 2000, 12, 2247-2258. [CrossRef] [PubMed]

66. Knight, H.; Trewavas, A.J.; Knight, M.R. Calcium signalling in Arabidopsis thaliana responding to drought and salinity. Plant J. 1997, 12, 1067-1078. [CrossRef] [PubMed]

67. Huang, P.; Ju, H.W.; Min, J.H.; Zhang, X.; Kim, S.H.; Yang, K.Y.; Kim, C.S. Overexpression of L-type lectin-like protein kinase 1 confers pathogen resistance and regulates salinity response in Arabidopsis thaliana. Plant Sci. 2013, 203, 98-106. [CrossRef] [PubMed]

68. Xu, Y.; Zhan, C.; Huang, B. Heat shock proteins in association with heat tolerance in grasses. Int. J. Proteom. 2011, 2011, 529648. [CrossRef] [PubMed]

69. Sun, X.; Sun, C.; Li, Z.; Hu, Q.; Han, L.; Luo, H. AsHSP17, a creeping bentgrass small heat shock protein modulates plant photosynthesis and ABA-dependent and independent signalling to attenuate plant response to abiotic stress. Plant Cell Environ. 2016, 39, 1320-1337. [CrossRef] [PubMed]

70. Swindell, W.R.; Huebner, M.; Weber, A.P. Transcriptional profiling of Arabidopsis heat shock proteins and transcription factors reveals extensive overlap between heat and non-heat stress response pathways. BMC Genom. 2007, 8, 125. [CrossRef] [PubMed]

71. Perlin, M.H.; Andrews, J.; Toh, S.S. Essential letters in the fungal alphabet: ABC and MFS transporters and their roles in survival and pathogenicity. Adv. Genet. 2014, 85, 201-253. [PubMed]

72. Kim, D.Y.; Jin, J.Y.; Alejandro, S.; Martinoia, E.; Lee, Y. Overexpression of AtABCG36 improves drought and salt stress resistance in Arabidopsis. Physiol. Plant 2010, 139, 170-180. [CrossRef] [PubMed]

73. Chan, C.; Lam, H.M. A putative $\lambda$ class glutathione S-transferase enhances plant survival under salinity stress. Plant Cell Physiol. 2014, 55, 570-579. [CrossRef] [PubMed]

74. Le Martret, B.; Poage, M.; Shiel, K.; Nugent, G.D.; Dix, P.J. Tobacco chloroplast transformants expressing genes encoding dehydroascorbate reductase, glutathione reductase, and glutathione-S-transferase, exhibit altered anti-oxidant metabolism and improved abiotic stress tolerance. Plant Biotechnol. J. 2011, 9, 661-673. [CrossRef] [PubMed]

75. Liu, D.; Liu, Y.; Rao, J.; Wang, G.; Li, H.; Ge, F.; Chen, C. Overexpression of the glutathione S-transferase gene from Pyrus pyrifolia fruit improves tolerance to abiotic stress in transgenic tobacco plants. Mol. Biol. 2013, 47, 591-601. [CrossRef]

76. Gou, X.; Yin, H.; He, K.; Du, J.; Yi, J.; Xu, S.; Lin, H.; Clouse, S.D.; Li, J. Genetic evidence for an indispensable role of somatic embryogenesis receptor kinases in brassinosteroid signaling. PLoS Genet. 2012, 8, e1002452. [CrossRef] [PubMed] 
77. Bai, M.Y.; Zhang, L.Y.; Gampala, S.S.; Zhu, S.W.; Song, W.Y.; Chong, K.; Wang, Z.Y. Functions of OsBZR1 and 14-3-3 proteins in brassinosteroid signaling in rice. Proc. Natl. Acad. Sci. USA 2007, 104, 13839-13844. [CrossRef] [PubMed]

78. Deinlein, U.; Stephan, A.B.; Horie, T.; Luo, W.; Xu, G.; Schroeder, J.I. Plant salt-tolerance mechanisms. Trends Plant Sci. 2014, 19, 371-379. [CrossRef] [PubMed]

79. Sun, R.; Ye, R.; Gao, L.; Zhang, L.; Wang, R.; Mao, T.; Zheng, Y.; Li, D.; Lin, Y. Characterization and Ectopic Expression of CoWRI1, an AP2/EREBP Domain-Containing Transcription Factor from Coconut (Cocos nucifera L.) Endosperm, Changes the Seeds Oil Content in Transgenic Arabidopsis thaliana and Rice (Oryza sativa L.). Front. Plant Sci. 2017, 8, 63. [CrossRef] [PubMed]

80. Jeong, J.S.; Kim, Y.S.; Baek, K.H.; Jung, H.; Ha, S.H.; Do Choi, Y.; Kim, M.; Reuzeau, C.; Kim, J.K. Root-specific expression of OsNAC10 improves drought tolerance and grain yield in rice under field drought conditions. Plant Physiol. 2010, 153, 185-197. [CrossRef] [PubMed]

81. Mittler, R.; Kim, Y.; Song, L.; Coutu, J.; Coutu, A.; Ciftci-Yilmaz, S.; Lee, H.; Stevenson, B.; Zhu, J.K. Gain- and loss-of-function mutations in Zat10 enhance the tolerance of plants to abiotic stress. FEBS Lett. 2006, 580, 6537-6542. [CrossRef] [PubMed]

82. Nozue, K.; Covington, M.F.; Duek, P.D.; Lorrain, S.; Fankhauser, C.; Harmer, S.L.; Maloof, J.N. Rhythmic growth explained by coincidence between internal and external cues. Nature 2007, 448, 358-361. [CrossRef] [PubMed]

83. Harmer, S.L. The circadian system in higher plants. Annu. Rev. Plant Biol. 2009, 60, 357-377. [CrossRef] [PubMed]

84. Gehan, M.A.; Greenham, K.; Mockler, T.C.; McClung, C.R. Transcriptional networks-crops, clocks, and abiotic stress. Curr. Opin. Plant Bio.l 2015, 24, 39-46. [CrossRef] [PubMed]

85. Kim, W.Y.; Ali, Z.; Park, H.J.; Park, S.J.; Cha, J.Y.; Perez-Hormaeche, J.; Quintero, F.J.; Shin, G.; Kim, M.R.; Qiang, Z.; et al. Release of SOS2 kinase from sequestration with GIGANTEA determines salt tolerance in Arabidopsis. Nat. Commun. 2013, 4, 1352. [CrossRef] [PubMed]

86. Li, S.; Yue, W.; Wang, M.; Qiu, W.; Zhou, L.; Shou, H. Mutation of OsGIGANTEA Leads to Enhanced Tolerance to Polyethylene Glycol-Generated Osmotic Stress in Rice. Front. Plant Sci. 2016, 7, 465. [CrossRef] [PubMed]

(C) 2017 by the authors. Licensee MDPI, Basel, Switzerland. This article is an open access article distributed under the terms and conditions of the Creative Commons Attribution (CC BY) license (http://creativecommons.org/licenses/by/4.0/). 